\title{
ON THE WKB METHOD FOR CERTAIN THIRD ORDER ORDINARY DIFFERENTIAL EQUATIONS
}

\author{
Dedicated to the 60th birthday of Prof. Toshihusa Kimura \\ By Minoru NaKano, Masatoshi Namiki \\ AND TOSHIHIKo NISHIMOTO
}

\section{§1. Introduction}

In this paper, we consider the so-called WKB type asymptotic expansions of solutions of certain third order linear ordinary differential equations in the whole complex $x$-plane. The equations we discuss are of the form

$$
\varepsilon^{3} y^{\prime \prime \prime}+\varepsilon^{2} p_{1}(x) y^{\prime \prime}+\varepsilon p_{2}(x) y^{\prime}+p_{3}(x) y=0,
$$

where $p_{j}(x)(j=1,2,3)$ are at this moment assumed to be entire functions of $x$ but in later sections polynomials or linear functions of $x$, and $\varepsilon$ is a small positive parameter.

The characteristic equation for (1.1) is defined by

$$
k(\lambda, x) \equiv \lambda^{3}+p_{1}(x) \lambda^{2}+p_{2}(x) \lambda+p_{3}(x)=0
$$

and the roots of the above equation are called the characteristic roots of (1.1) which we denote by $\lambda_{1}(x), \lambda_{2}(x)$ and $\lambda_{3}(x)$.

The points $x_{0}$ where at least two characteristic roots coincide

$$
\lambda_{j}\left(x_{0}\right)=\lambda_{k}\left(x_{0}\right) \quad(\jmath \neq k)
$$

are called turning points of the equation (1.1). We define $(j, k)$-Stokes curves and $(j, k)$-anti Stokes curves by

$$
\begin{aligned}
& \operatorname{Re} \int_{x_{0}}^{x}\left\{\lambda_{j}(t)-\lambda_{k}(t)\right\} d t=0, \\
& \operatorname{Im} \int_{x_{0}}^{x}\left\{\lambda_{j}(t)-\lambda_{k}(t)\right\} d t=0
\end{aligned}
$$

respectively, if there exists a turning point $x_{0}$ satisfying $\lambda_{j}\left(x_{0}\right)=\lambda_{k}\left(x_{0}\right)$.

The object of the WKB theory is to develop a set of rules for continuing

Received July 5, 1990; revised April 1, 1991. 
asymptotic solutions to all regions of the complex plane. For the second order ordinary differential equations, the theory has been successfully developed by many authers, for examples Evgrafov and Fedoryuk [2], Heading [5], Fröman and Fröman [3], Olver [6] and Voros [8]. On the contrary, the theory for higher order cases is seemed to be quite incomplete, and most papers have concerned with the behavior of solutions in the small neighborhood of an isolated turning point, or constructing asymptotic expansions as $x$ tends to infinity, that is, in a neighborhood of infinity, Paris and Wood [7].

Recently Berk, Nevines and Roberts [1] gave a first step to the global WKB analysis for higher order cases. The most remarkable assertion in that paper is that under some assumptions the existence of secondary Stokes curves is demonstrated. The secondary Stokes curve issues from a crossing point of two anti Stokes curves and along them the so-called Stokes phenomenon occurs. Here we have to notice that for the second order ordinary differential equations the Stokes curves (or the anti Stokes curves) cross only at turning points but for the higher order cases two Stokes curves (or anti Stokes curves) can cross at ordinary points (not turning points) as shown in a simple example, see Fig. 4.1.

To construct the WKB theory for equations of order $n \geqq 3$ seems to be quite difficult in general, Fedoryuk [4], then we start our studies of third order ordinary differential equations by treating examples which can be analyzed rigorously. Comparing with the WKB theory of second order equations, we consider about several questions such as (1) Stokes curve configuration (2) existence of admissible domains and (3) the connection rules in the whole complex $x$-plane.

The reasons why we focus upon the studies of the WKB theory for third order ordinary diffential equations instead of considering those for general higher order ordinary differential equations are as follows. Firstly we can always calculate formal solutions of WKB type of (1.1) (given in the section 2) by using the Cardano's formula to obtain $\lambda_{j}(x)$, and secondly the characteristic difficulties such as Stokes curve configuration and connection rules in the whole complex plane for higher order equations are present even in the third order ordinary differential equations.

This paper consists of six sections. In the section 2, we describe selfcontainedly how to get formal solutions of (1.1). Firstly we transform the equation (1.1) into a system of three differential equations and by using a successive diagonalization technique we obtain formal solutions which we call "formal WKB solutions". An existence theorem is also given.

In the section 3 , we assume that the coefficients $p_{j}(x)$ of the equation (1.1) are linear functions of $x$. Then it is possible to express solutions of (1.1) by the Laplace integral, and asymptotic expansions of solutions as $\varepsilon$ tends to zero may be obtained by the so-called saddle point method or the method of steepest descent. In this analysis, the independent variable $x$ is contained as another parameter in the asymptotic expansions which we compare with formal WKB 
soluions. The connection formulas or rules between solutions defined in different regions can be obtained by applying the Cauchy's integral theorem of analytic functions to the Laplace integral representation of solutions.

In the sections 4 and 5 , we consider three examples which illustrate the methods. Each example can be considered as a variant of the Airy function or the parabolic cylinder function in a sense but has different aspects.

In the section 6 we give several interesting examples to be considered. We expect that these examples can be exploited as suitable related equations for more complicated equations of similar natures, as the Airy equation plays in the second order equations.

\section{§2. The WKB solutions}

In this section, we restrict ourselves to the case when all the coefficients $p_{j}(x)$ are polynomials.

THEOREM 2.1. The differential equation (1.1) possesses formal WKB solutions to the order $\varepsilon \tilde{y}_{j}(x, \varepsilon)$ of the following form.

$$
\tilde{y}_{j}(x, \varepsilon)=A, \exp \left(\frac{1}{\varepsilon} \int_{x_{0}}^{x}\left\{\lambda_{j}(s)+\varepsilon \lambda_{j}^{(1)}(s)\right\} d s\right) \quad(j=1,2,3),
$$

where $\lambda_{j}{ }^{(1)}(x)$ is given by (2.8), and $x_{0}$ and $A$, are constants.

Proof. Introducing dependent variables $y^{(j)}$ by

$$
y^{(1)}=y, \quad y^{(2)}=\varepsilon y^{\prime}, \quad y^{(3)}=\varepsilon^{2} y^{\prime \prime}
$$

the differential equation (1.1) becomes a system of differential equations

$$
\varepsilon \frac{d Y}{d x}=A(x) Y
$$

where

$$
Y=\left(\begin{array}{l}
y^{(1)} \\
y^{(2)} \\
y^{(3)}
\end{array}\right), \quad A=\left(\begin{array}{rrr}
0 & 1 & 0 \\
0 & 0 & 1 \\
-p_{3} & -p_{2} & -p_{1}
\end{array}\right) .
$$

The equation $\operatorname{det}(\lambda E-A)=0$ is the characteristic equation

$$
\lambda^{3}+p_{1}(x) \lambda^{2}+p_{2}(x) \lambda+p_{3}(x)=0 .
$$

By using the Cardano's formula, the roots $\lambda_{j}(x)(j=1,2,3)$ are obtained as follows :

$$
\begin{gathered}
\lambda_{j}=\xi_{j}-\frac{p_{1}}{3} \\
\xi_{1}=\sqrt[3]{\alpha}+\sqrt[3]{\beta}, \quad \xi_{2}=\omega \cdot \sqrt[3]{\alpha}+\omega^{2} \cdot \sqrt[3]{\beta}, \quad \xi_{3}=\omega^{2} \cdot \sqrt[3]{\alpha}+\omega \cdot \sqrt[3]{\beta}
\end{gathered}
$$


where

and

$$
\omega=\exp (2 \pi i / 3)
$$

$$
\alpha=\frac{-q+\sqrt{q^{2}+4 p^{3}}}{2}, \quad \beta=\frac{-q-\sqrt{q^{2}+4 p^{3}}}{2}
$$

with

$$
\begin{gathered}
p(x)=\frac{3 p_{2}(x)-p_{1}(x)^{2}}{9} \\
q(x)=\frac{2 p_{1}(x)^{3}-9 p_{1}(x) p_{2}(x)+27 p_{3}(x)}{27} .
\end{gathered}
$$

By a linear transformation

$$
Y=P(x) U_{1}, \quad P(x)=\left(\begin{array}{ccc}
1 & 1 & 1 \\
\lambda_{1}(x) & \lambda_{2}(x) & \lambda_{3}(x) \\
\lambda_{1}(x)^{2} & \lambda_{2}(x)^{2} & \lambda_{3}(x)^{2}
\end{array}\right)
$$

the equation (2.1) is changed into

$$
\varepsilon \frac{d U_{1}}{d x}=B(x, \varepsilon) U_{1}
$$

where

$$
B(x, \varepsilon)=P(x)^{-1} A(x) P(x)-\varepsilon P(x)^{-1} P^{\prime}(x)
$$

with

$$
P(x)^{-1} A(x) P(x)=\left(\begin{array}{ccc}
\lambda_{1}(x) & 0 & 0 \\
0 & \lambda_{2}(x) & 0 \\
0 & 0 & \lambda_{3}(x)
\end{array}\right) \equiv \Lambda(x)
$$

and

$$
P(x)^{-1} P^{\prime}(x)=\left(\begin{array}{lll}
\frac{2 \lambda_{1}-\left(\lambda_{2}+\lambda_{3}\right)}{\left(\lambda_{1}-\lambda_{2}\right)\left(\lambda_{1}-\lambda_{3}\right)} \lambda_{1}{ }^{\prime} & \frac{2 \lambda_{2}-\left(\lambda_{2}+\lambda_{3}\right)}{\left(\lambda_{1}-\lambda_{2}\right)\left(\lambda_{1}-\lambda_{3}\right)} \lambda_{2}{ }^{\prime} & \frac{2 \lambda_{3}-\left(\lambda_{2}+\lambda_{3}\right)}{\left(\lambda_{1}-\lambda_{2}\right)\left(\lambda_{1}-\lambda_{3}\right)} \lambda_{3}{ }^{\prime} \\
\frac{2 \lambda_{1}-\left(\lambda_{1}+\lambda_{3}\right)}{\left(\lambda_{2}-\lambda_{1}\right)\left(\lambda_{2}-\lambda_{3}\right)} \lambda_{1}{ }^{\prime} & \frac{2 \lambda_{2}-\left(\lambda_{1}+\lambda_{3}\right)}{\left(\lambda_{2}-\lambda_{1}\right)\left(\lambda_{2}-\lambda_{3}\right)} \lambda_{2}{ }^{\prime} & \frac{2 \lambda_{3}-\left(\lambda_{1}+\lambda_{3}\right)}{\left(\lambda_{2}-\lambda_{1}\right)\left(\lambda_{2}-\lambda_{3}\right)} \lambda_{3}{ }^{\prime} \\
\frac{2 \lambda_{1}-\left(\lambda_{1}+\lambda_{2}\right)}{\left(\lambda_{3}-\lambda_{1}\right)\left(\lambda_{3}-\lambda_{2}\right)} \lambda_{1}{ }^{\prime} & \frac{2 \lambda_{2}-\left(\lambda_{1}+\lambda_{2}\right)}{\left(\lambda_{3}-\lambda_{1}\right)\left(\lambda_{3}-\lambda_{2}\right)} \lambda_{2}{ }^{\prime} & \frac{2 \lambda_{3}-\left(\lambda_{1}+\lambda_{2}\right)}{\left(\lambda_{3}-\lambda_{1}\right)\left(\lambda_{3}-\lambda_{2}\right)} \lambda_{3}{ }^{\prime}
\end{array}\right) .
$$

Hereafter we construct successively linear transformations so that the matrix $B(x, \varepsilon)$ becomes formal power series of $\varepsilon$ with diagonal coefficients. To make the coefficient matrix of $\varepsilon$ diagonal, for example, we change (2.5) by a transformation

$$
U_{1}=\left(E+\varepsilon Q_{1}(x)\right) U_{2},
$$


where $E$ is the unit matrix of order 3 and $Q_{1}(x)$ is to be taken appropriately later. Then we have

$$
\begin{aligned}
\varepsilon \frac{d U_{2}}{d x} & =\left(E+\varepsilon Q_{1}\right)^{-1}\left\{\left(\Lambda-\varepsilon P^{-1} P^{\prime}\right)\left(E+\varepsilon Q_{1}\right)-\varepsilon^{2} Q_{1}{ }^{\prime}\right\} U_{2} \\
& =\left\{\Lambda+\varepsilon\left(\Lambda Q_{1}-Q_{1} \Lambda-P^{-1} P^{\prime}\right)+O\left(\varepsilon^{2}\right)\right\} U_{2} .
\end{aligned}
$$

Here the matrix $Q_{1}$ can be chosen so that the matrix $\left(\Lambda Q_{1}-Q_{1} \Lambda-P^{-1} P^{\prime}\right)$ diagonal. Indeed, let elements of the matrix $Q_{1}$ be $q_{j k}$, then after a short calculation we have

We choose $q_{j k}$ so that

$$
\Lambda Q_{1}-Q_{1} \Lambda=\left(\begin{array}{ccc}
0 & \left(\lambda_{1}-\lambda_{2}\right) q_{12} & \left(\lambda_{1}-\lambda_{3}\right) q_{13} \\
\left(\lambda_{2}-\lambda_{1}\right) q_{21} & 0 & \left(\lambda_{2}-\lambda_{3}\right) q_{23} \\
\left(\lambda_{3}-\lambda_{1}\right) q_{31} & \left(\lambda_{3}-\lambda_{2}\right) q_{32} & 0
\end{array}\right) .
$$

that is

$$
\Lambda Q_{1}-Q_{1} \Lambda-P^{-1} P^{\prime}=-\operatorname{diag}\left[P^{-1} P^{\prime}\right],
$$

$$
Q_{1}=\left(\begin{array}{ccc}
0 & \frac{2 \lambda_{2}-\left(\lambda_{2}+\lambda_{3}\right)}{\left(\lambda_{1}-\lambda_{3}\right)\left(\lambda_{1}-\lambda_{2}\right)^{2}} \lambda_{2}{ }^{\prime} & \frac{2 \lambda_{3}-\left(\lambda_{2}+\lambda_{3}\right)}{\left(\lambda_{1}-\lambda_{2}\right)\left(\lambda_{1}-\lambda_{3}\right)^{2}} \lambda_{3}{ }^{\prime} \\
\frac{2 \lambda_{1}-\left(\lambda_{1}+\lambda_{3}\right)}{\left(\left(\lambda_{2}-\lambda_{3}\right) \lambda_{2}-\lambda_{1}\right)^{2}} \lambda_{1}{ }^{\prime} & 0 & \frac{2 \lambda_{3}-\left(\lambda_{1}+\lambda_{3}\right)}{\left(\lambda_{2}-\lambda_{1}\right)\left(\lambda_{2}-\lambda_{3}\right)^{2}} \lambda_{3}{ }^{\prime} \\
\frac{2 \lambda_{1}-\left(\lambda_{1}+\lambda_{2}\right)}{\left(\lambda_{3}-\lambda_{2}\right)\left(\lambda_{3}-\lambda_{1}\right)^{2}} \lambda_{1}{ }^{\prime} & \frac{2 \lambda_{2}-\left(\lambda_{1}+\lambda_{2}\right)}{\left(\lambda_{3}-\lambda_{1}\right)\left(\lambda_{3}-\lambda_{2}\right)^{2}} \lambda_{2}{ }^{\prime} & 0
\end{array}\right) .
$$

Here $\operatorname{diag} A$ means that it is a diagonal matrix whose diagonal elements are the diagonal elements of the matrix $A$. Then by (2.6), the differential equation (2.5) becomes

$$
\varepsilon \frac{d U_{2}}{d x}=\left\{\Lambda(x)+\varepsilon \Lambda_{1}(x)+\varepsilon^{2} C(x, \varepsilon)\right\} U_{2},
$$

where $\Lambda_{1}(x)=\operatorname{diag}\left\{\lambda_{1}{ }^{(1)}, \lambda_{2}{ }^{(1)}, \lambda_{3}{ }^{(1)}\right\}$ with

$$
\begin{aligned}
& \lambda_{1}{ }^{(1)}(x)=-\frac{2 \lambda_{1}-\left(\lambda_{2}+\lambda_{3}\right)}{\left(\lambda_{1}-\lambda_{2}\right)\left(\lambda_{1}-\lambda_{3}\right)} \lambda_{1}=-\left\{\frac{\lambda_{1}{ }^{\prime}}{\lambda_{1}-\lambda_{2}}+\frac{\lambda_{1}{ }^{\prime}}{\lambda_{1}-\lambda_{3}}\right\}, \\
& \lambda_{2}{ }^{(1)}(x)=-\frac{2 \lambda_{2}-\left(\lambda_{1}+\lambda_{3}\right)}{\left(\lambda_{2}-\lambda_{1}\right)\left(\lambda_{2}-\lambda_{3}\right)} \lambda_{2}{ }^{\prime}=-\left\{\frac{\lambda_{2}{ }^{\prime}}{\lambda_{2}-\lambda_{1}}+\frac{\lambda_{2}{ }^{\prime}}{\lambda_{2}-\lambda_{3}}\right\}, \\
& \lambda_{3}{ }^{(1)}(x)=-\frac{2 \lambda_{3}-\left(\lambda_{1}+\lambda_{2}\right)}{\left(\lambda_{3}-\lambda_{1}\right)\left(\lambda_{3}-\lambda_{2}\right)} \lambda_{3}{ }^{\prime}=-\left\{\frac{\lambda_{3}{ }^{\prime}}{\lambda_{3}-\lambda_{1}}+\frac{\lambda_{3}{ }^{\prime}}{\lambda_{3}-\lambda_{2}}\right\}
\end{aligned}
$$

and 
WKB METHOD FOR CERTAIN THIRD ORDER EQUATIONS

$$
\begin{aligned}
C(x, \varepsilon)= & Q_{1} P^{-1} P^{\prime}-P^{-1} P^{\prime} Q_{1}-Q_{1} \Lambda Q_{1}-Q_{1}{ }^{\prime}+\left(E+\varepsilon Q_{1}\right)^{-1}{Q_{1}}^{2} \Lambda \\
& +\varepsilon\left\{Q_{1} P^{-1} P^{\prime} Q_{1}+Q_{1} Q_{1}{ }^{\prime}+\left(E+\varepsilon Q_{1}\right)^{-1} Q_{1}{ }^{2}\left(\Lambda Q_{1}-P^{-1} P^{\prime}\right)\right\} \\
& -\varepsilon^{2}\left(E+\varepsilon Q_{1}\right)^{-1} Q_{1}{ }^{2}\left(P^{-1} P^{\prime} Q_{1}+Q_{1}{ }^{\prime}\right) .
\end{aligned}
$$

Further we can make a transformation of the form

$$
U_{2}=\left\{E+\varepsilon^{2} Q_{2}(x)\right\} U_{3},
$$

by which the coefficient matrix of $\varepsilon^{2}$ of (2.7) becomes diagonal, and so on.

The diagonal elements $\lambda_{j}{ }^{(1)}(x)$ of $\Lambda_{1}(x)$ can be written in other ways. Since

$$
\lambda_{j}^{(1)}(x)=-\frac{1}{2} \lambda_{j}{ }^{\prime}(x) \frac{k_{\lambda \lambda}\left(\lambda_{j}(x), x\right)}{k_{\lambda}\left(\lambda_{j}(x), x\right)}
$$

and

$$
\left\{\left(\lambda_{1}-\lambda_{2}\right)\left(\lambda_{1}-\lambda_{3}\right)\right\}^{\prime}=2\left\{2 \lambda_{1}-\left(\lambda_{2}+\lambda_{3}\right)\right\} \lambda_{1}{ }^{\prime}+p_{2}{ }^{\prime}+2 \lambda_{1} p_{1}{ }^{\prime}
$$

we have from (2.8)

$$
\lambda_{1}{ }^{(1)}(x)=-\frac{\left\{\left(\lambda_{1}-\lambda_{2}\right)\left(\lambda_{1}-\lambda_{3}\right)\right\}^{\prime}}{2\left(\lambda_{1}-\lambda_{2}\right)\left(\lambda_{1}-\lambda_{3}\right)}+\frac{p_{2}{ }^{\prime}+2 \lambda_{1} p_{1}{ }^{\prime}}{2\left(\lambda_{1}-\lambda_{2}\right)\left(\lambda_{1}-\lambda_{3}\right)} .
$$

Analogously,

$$
\begin{aligned}
& \lambda_{2}{ }^{(1)}(x)=-\frac{\left\{\left(\lambda_{2}-\lambda_{1}\right)\left(\lambda_{2}-\lambda_{3}\right)\right\}^{\prime}}{2\left(\lambda_{2}-\lambda_{1}\right)\left(\lambda_{2}-\lambda_{3}\right)}+\frac{p_{2}{ }^{\prime}+2 \lambda_{2} p_{1}{ }^{\prime}}{2\left(\lambda_{2}-\lambda_{1}\right)\left(\lambda_{2}-\lambda_{3}\right)}, \\
& \lambda_{3}{ }^{(1)}(x)=-\frac{\left\{\left(\lambda_{3}-\lambda_{1}\right)\left(\lambda_{3}-\lambda_{2}\right)\right\}^{\prime}}{2\left(\lambda_{3}-\lambda_{1}\right)\left(\lambda_{3}-\lambda_{2}\right)}+\frac{p_{2}{ }^{\prime}+2 \lambda_{3} p_{1}{ }^{\prime}}{2\left(\lambda_{3}-\lambda_{1}\right)\left(\lambda_{3}-\lambda_{2}\right)} .
\end{aligned}
$$

Let $U_{2}(x, \varepsilon)$ be a fundamental system of solutions of the equation (2.7) to the order of $\varepsilon$, that is

$$
U_{2}(x, \varepsilon)=\exp \left(\int\left\{\frac{\Lambda(s)}{\varepsilon}+\Lambda_{1}(s)\right\} d s\right) .
$$

By the transformation (2.4) and (2.6), we obtain the matrix $Y(x, \varepsilon)$ which is the fundamental system of solutions of the equation (2.1) to the order of $\varepsilon$, and then the elements $\tilde{y}_{j}$ of the first row of $Y(x, \varepsilon)$ satisfy the differential equation (1.1) to the order of $\varepsilon$.

Here we put

$$
\begin{array}{r}
\tilde{y}_{j}(x, \varepsilon)=A_{j} \exp \left(\frac{1}{\varepsilon} \int_{x_{0}}^{x}\left\{\lambda_{j}(s)+\varepsilon \lambda_{j}{ }^{(1)}(s)\right\} d s\right), \\
\left(\jmath=1,2,3 ; x_{0}, A=\text { const. }\right) .
\end{array}
$$

We call these functions $\tilde{y}_{j}(x, \varepsilon)$ "formal WKB solutions" of the equation (1.1). If ${p_{1}}^{\prime}={p_{2}}^{\prime}=0$, from $\left(2.8^{\prime}\right)$ the expression of $\tilde{y}_{j}(x, \varepsilon)$ becomes 


$$
\tilde{y}_{j}(x, \varepsilon)=\frac{A_{j}}{\sqrt{\left(\lambda_{j}-\lambda_{k}\right)\left(\lambda_{j}-\lambda_{l}\right)}} \exp \frac{1}{\varepsilon} \int_{x_{0}}^{x} \lambda_{j}(s) d s,
$$

and then the expressions $(2.10)$ and $\left(2.10^{\prime}\right)$ can be considered as a generalization of the WKB solutions of the second order ordinary differential equations. Q.E.D.

It is possible to prove the existence theorem: corresponding to each formal WKB solution there exists a true solution of (1.1) in a certain ragion of the $x$-plane whose asymptotic expansion as $\varepsilon$ tends to zero coincides with the formal WKB solution.

Let $D$ be a simply-connected and unbounded domain in the complex $x$-plane, and suppose that there are no turning points of (1.1) in $D$. We introduce the notation

$$
\begin{aligned}
& \xi_{j}\left(x_{0}, x\right)=\int_{x_{0}}^{x} \lambda_{j}(s) d s, \\
& \xi_{j k}\left(x_{0}, x\right)=\xi_{j}\left(x_{0}, x\right)-\xi_{k}\left(x_{0}, x\right) \quad(j, k=1,2,3, j \neq k) .
\end{aligned}
$$

Let the curve $\gamma_{j k}(x)$ lie in $D$ which joins the point $x$ and $\infty$, and suppose that $\operatorname{Re} \xi_{j k}(t, x)$ increases when $t \in \gamma_{j k}(x)$ moves from $x$ to $\infty$. Then the curve $\gamma_{j k}(x)$ is called a $(j, k)$-canonical path.

$A$ set $\Gamma_{j}(x)=\left(\gamma_{j_{1}}(x), \gamma_{j 2}(x), \gamma_{j 3}(x)\right)$ consisting of $(j, k)$-canonical paths $(k=$ $1,2,3)$ is called a $j$-canonical vector path. $D$ is called a $\lambda_{j}$-admissible domain if for each $x \in D$ there is a $j$-canonical vector path $\Gamma_{j}(x)$. Then we can prove the following existence theorem by the usual method, for example, by the method of successive approximation.

THEOREM 2.2. We assume that $D$ is a $\lambda_{j}$-admissible domain.

Then the equation (1.1) has a solution $y_{j}(x, \varepsilon)$ of the form

$$
y_{j}(x, \varepsilon)=\exp \left\{\frac{1}{\varepsilon} \int_{x_{0}}^{x} \lambda_{j}(t) d t+\int_{x_{0}}^{x} \lambda_{j}{ }^{(1)}(t) d t\right\}\left[1+\varepsilon \delta_{j}(x, \varepsilon)\right],
$$

where for $0<\varepsilon \leqq \varepsilon_{0}$

$$
\left|\delta_{j}(x, \varepsilon)\right| \leqq \rho_{j}(x), \quad \lim _{x \rightarrow \infty, x \in D} \rho_{j}(x)=0 \quad(j=1,2,3) .
$$

Outline of proof. The differential equation (1.1) is transformed into the differential equation (2.7) of the system type after some transformations (2.4), (2.6) and so on. We are proving the theorem of the case for $j=1$.

Let

$$
U_{2}=\exp \left(\frac{1}{\varepsilon} \int^{x}\left\{\lambda_{1}(s)+\varepsilon \lambda_{1}{ }^{(1)}(s)\right\} d s\right) \cdot U, \quad U=\left(u_{1}, u_{2}, u_{3}\right)^{t},
$$

then the equation (2.7) becomes 


$$
\varepsilon \frac{d U}{d x}=\left\{\left(\begin{array}{ccc}
0 & 0 & 0 \\
0 & \lambda_{2}-\lambda_{1} & 0 \\
0 & 0 & \lambda_{3}-\lambda_{1}
\end{array}\right)+\varepsilon\left(\begin{array}{ccc}
0 & 0 & 0 \\
0 & \lambda_{2}{ }^{(1)}-\lambda_{1}{ }^{(1)} & 0 \\
0 & 0 & \lambda_{3}{ }^{(1)}-\lambda_{1}{ }^{(1)}
\end{array}\right)+\varepsilon^{2} C\right\} U .
$$

The equation (2.13) is equivalent to the integral equation

$$
\begin{aligned}
U(x, \varepsilon)= & U_{0}(x, \varepsilon) K \\
& +\varepsilon \int_{\Gamma_{1}} U_{0}(x, \varepsilon) U_{0}^{-1}(t, \varepsilon) C(t, \varepsilon) U(t, \varepsilon) d t,
\end{aligned}
$$

where $U_{0}(x, \varepsilon)\left(U_{0}\left(x_{0}, \varepsilon\right)=E\right)$ is the diagonal matrix solution of

$$
\varepsilon \frac{d U_{0}}{d x}=\left\{\operatorname{diag}\left(0, \lambda_{2}-\lambda_{1}+\varepsilon\left(\lambda_{2}{ }^{(1)}-\lambda_{1}{ }^{(1)}\right), \lambda_{3}-\lambda_{1}+\varepsilon\left(\lambda_{3}{ }^{(1)}-\lambda_{1}{ }^{(1)}\right)\right\} U_{0},\right.
$$

$K$ is an arbitrary constant vector and $\Gamma_{1}$ is a 1 -canonical vector path $\Gamma_{1}=$ $\left(\gamma_{11}, \gamma_{12}, \gamma_{13}\right)$ in a $\lambda_{1}$-admissible domain $D$.

We can get, as follows, the solution of the equation (2.14) by the successive approximation defined by

$$
\begin{aligned}
& U^{(0)}(x, \varepsilon)=U_{0}(x, \varepsilon) K, \\
& U^{(n+1)}(x, \varepsilon)=U_{0}(x, \varepsilon) K \\
& +\varepsilon \int_{\Gamma_{1}} U_{0}(x, \varepsilon) U_{0}^{-1}(t, \varepsilon) C(t, \varepsilon) U^{(n)}(t, \varepsilon) d t \\
& (n=0,1,2,3, \cdots) \text {. }
\end{aligned}
$$

If we put

and

$$
K=(1,0,0)^{t}, \quad U^{(n)}=\left(u_{1}{ }^{(n)}, u_{2}{ }^{(n)}, u_{3}{ }^{(n)}\right)^{t},
$$

$$
C=\left(C_{p q}\right) \text {, }
$$

then the equations (2.15) are equivalent to

$$
\left(\begin{array}{l}
u_{1}{ }^{(0)} \\
u_{2}{ }^{(0)} \\
u_{3}{ }^{(0)}
\end{array}\right)=\left(\begin{array}{l}
1 \\
0 \\
0
\end{array}\right)
$$

$$
\left(\begin{array}{l}
u_{1}{ }^{(n+1)} \\
u_{2}{ }^{(n+1)} \\
u_{3}{ }^{(n+1)}
\end{array}\right)=\left(\begin{array}{l}
1+\varepsilon \int_{\gamma_{11}} \sum_{q=1}^{3} C_{1 q} u_{q}{ }^{(n)} d t \\
\varepsilon \int_{\gamma_{12}} \exp \left\{\int_{t}^{x}\left(\frac{\lambda_{2}-\lambda_{1}}{\varepsilon}+\lambda_{2}{ }^{(1)}-\lambda_{1}{ }^{(1)}\right) d s\right\} \sum_{q=1}^{3} C_{2 q} u_{q}{ }^{(n)} d t \\
\varepsilon \int_{\gamma_{13}} \exp \left\{\int_{t}^{x}\left(\frac{\lambda_{3}-\lambda_{1}}{\varepsilon}+\lambda_{3}{ }^{(1)}-\lambda_{1}{ }^{(1)}\right) d s\right\} \sum_{q=1}^{3} C_{3 q} u_{q}{ }^{(n)} d t
\end{array}\right) .
$$


As all coefficients $p_{k}(x)$ of the equation (1.1) are polynomial, the characteristic roots $\lambda_{k}$ and $\lambda_{k}{ }^{(1)}(k=1,2,3)$ have following properties in the $\lambda_{1}$-admissible domain $D$ : along $\gamma_{1 k}(k=2,3)$

$$
\operatorname{Re} \int_{t}^{x}\left\{\lambda_{k}-\lambda_{1}\right\} d s=-\operatorname{Re} \xi_{1 k}(t, x) \leqq \delta_{0}<0,
$$

$$
\operatorname{Re}\left\{\int_{t}^{x}\left(\lambda_{k}-\lambda_{1}\right)\left\{1-\frac{\varepsilon}{\lambda_{k}-\lambda_{l}}\left(\lambda_{k}^{(1)}-\lambda_{1}^{(1)}\right)\right\} d s\right\} \leqq 0,
$$

and $\left|C_{p q}\right|(p, q=1,2,3)$ are bounded for $x$ in $D$ and for small $\varepsilon$, say, $0<\varepsilon$ $\leqq \varepsilon_{0}$, and that $C_{p q}=O\left(x^{-\nu-2}\right)(x \rightarrow \infty$ in $D)$ since all $\lambda_{k}=O\left(x^{\nu}\right)(x \rightarrow \infty$ in $D, \nu \geqq 0)$. After short calculation by using properties (2.17), we can get

$$
\begin{gathered}
\left|u_{q}{ }^{(n)}-u_{q}{ }^{(n)}\right| \leqq\left(3 \varepsilon \tilde{\delta}_{1}\right)^{n}, \\
\left|u_{q}{ }^{(n+1)}-u_{q}{ }^{(n)}\right| \leqq\left(3 \varepsilon \tilde{\delta}_{1}+1\right)\left(3 \varepsilon \tilde{\delta}_{1}\right)^{n+1}, \\
\tilde{\delta}_{1}(x, \varepsilon)=\inf _{r_{1 k}} \int_{r_{1 k}}\left|C_{p q}\right| d t \quad(p, q=1,2,3),
\end{gathered}
$$

where the inf is taken for any path $\gamma_{1 k}$ from $x$ to $\infty$ in $D$. From these inequalities, we get

$$
\begin{aligned}
\left|u_{q}-u_{q}^{(0)}\right| \leqq & \sum_{n=0}^{\infty}\left|u_{q}^{(n+1)}-u_{q}^{(n)}\right| \\
& \leqq \frac{3 \varepsilon \tilde{\delta}_{1}\left(1+3 \varepsilon \tilde{\delta}_{1}\right)}{1-3 \varepsilon \tilde{\delta}_{1}} \quad(q=1,2,3)
\end{aligned}
$$

and so $u_{q}=\lim _{n \rightarrow \infty} u_{q}{ }^{(n)}$ converges.

Thus $U=\left(u_{1}, u_{2}, u_{3}\right)^{t}$ is a solution of the integral equation (2.14). From the inequality (2.18), we have a relation

$$
u_{q}=u_{q}^{(0)}+\varepsilon \hat{\delta}_{1}(x, \varepsilon) \quad(q=1,2,3),
$$

where $\hat{\delta}_{1}$ is a bounded function for small $\varepsilon\left(0<\varepsilon \leqq \varepsilon_{0}\right)$ and $x \in D$. Put $\hat{\rho}_{1}(x)$ $=\sup _{0<\varepsilon \leq \varepsilon_{0}}\left|\hat{\delta}_{1}(x, \varepsilon)\right|$.

Letting $x \rightarrow \infty$ along the integral path $\gamma_{1 k}$ arbitrarily taken in the $\lambda_{1}$-admissible domain $D$

$$
\tilde{\delta}_{1} \longrightarrow 0,
$$

therefore $\hat{\rho}_{1}(x) \rightarrow 0(x \rightarrow \infty, x \in D)$.

From (2.19) we get

$$
u_{1}=1+\varepsilon \hat{\delta}_{1}(x, \varepsilon), \quad u_{q}=\varepsilon \hat{\delta}_{1}(x, \varepsilon) \quad\left(q=2,3,0<\varepsilon \leqq \varepsilon_{0}, x \in D\right) .
$$

By these relations and some transformations (2.12), (2.4), (2.6) etc., we can get the solution $y_{1}(x, \varepsilon)$ for $j=1$.

Q.E.D. 
The expression of the theorem 2.2 means the double asymptotic property of the solution $y_{j}(x, \varepsilon)$ :

$$
\begin{array}{ll}
y_{j}(x, \varepsilon) \sim \tilde{y}_{j}(x, \varepsilon) & (\varepsilon \rightarrow 0, x \in D), \\
y_{j}(x, \varepsilon) \sim \tilde{y}_{j}(x, \varepsilon) & \left(x \rightarrow \infty, x \in D, 0<\varepsilon \leqq \varepsilon_{0}\right) .
\end{array}
$$

We call the true solutions $y_{j}(x, \varepsilon)$ thus obtained the WKB solution of (1.1). But quite different from the case of second order equations, to construct a general theory specifying the admissible domain $D$ of the existence theorem is very difficult for the case of higher order equations even with polynomial coefficients. It is clear that the Stokes curves and anti Stokes curves play a fundamental role to characterize $D$.

If $x_{0}$ is a turning point such that $\lambda_{j}\left(x_{0}\right)=\lambda_{k}\left(x_{0}\right)(j \neq k)$, the level curves

$$
\begin{aligned}
& \operatorname{Re} \xi_{j k}\left(x_{0}, x\right)=\operatorname{Re} \int_{x_{0}}^{x}\left\{\lambda_{j}(t)-\lambda_{k}(t)\right\} d t=0, \\
& \operatorname{Im} \xi_{j k}\left(x_{0}, x\right)=\operatorname{Im} \int_{x_{0}}^{x}\left\{\lambda_{j}(t)-\lambda_{k}(t)\right\} d t=0
\end{aligned}
$$

issuing from $x_{0}$ are called $(j, k)$-Stokes curves and $(j, k)$-anti Stokes curves respectively.

The global structure of an individual Stokes curve and the whole Stokes curve configuration in the whole $x$-plane are very complicated. One reason is that the Stokes curves may intersect themselves at ordinary points (not turning points) since there are three independent functions $\xi_{j k}\left(x_{0}, x\right)(j, k=1,2,3 ; j>k)$ defining Stokes curves. The complicated expression (2.3) of the characteristic roots is also a serious obstacle of studying general properties of the Stokes curves.

It is possible to classify the equation (1.1) by means of the number of turning points and the order of merging of two characteristic roots.

Let the characteristic roots of $(1.1)$ be $\lambda_{j}(x)(\jmath=1,2,3)$, then a turning point $x_{0}$ where $\lambda_{j}\left(x_{0}\right)=\lambda_{k}\left(x_{0}\right)(j \neq k)$ is said to be simple if

$$
k_{\lambda \lambda}\left(\lambda_{j}\left(x_{0}\right), x_{0}\right) \neq 0
$$

and

$$
k_{x}\left(\lambda_{j}\left(x_{0}\right), x_{0}\right) \neq 0
$$

We consider in this paper the equations containing a simple turning point as well as multiple turning points which do not satisfy the condition (2.20) or (2.21), as examples in sections 4 and 5 . 


\section{§3. Expressions by the Laplace integral}

We assume in this section that the coefficients $p_{j}(x)$ are linear functions of $x: p_{j}(x)=p_{j 0}+p_{j 1} x(j=1,2,3)$. Then (1.1) becomes

$$
\varepsilon^{3} y^{\prime \prime \prime}+\varepsilon^{2}\left(p_{10}+p_{11} x\right) y^{\prime \prime}+\varepsilon\left(p_{20}+p_{21} x\right) y^{\prime}+\left(p_{30}+p_{31} x\right) y=0 \text {. }
$$

The solution $y(x, \varepsilon)$ of (3.1) may be expressed by the Laplace integral

$$
y(x, \varepsilon)=\int_{r} \phi(s, \varepsilon) \exp \left(\frac{x s}{\varepsilon}\right) d s .
$$

Substituting the above expression into (3.1), we can see that the function $y(x, \varepsilon)$ represented by (3.2) satisfies $(3.1)$ if $\phi(s, \varepsilon)$ and $\gamma$ satisfy

$$
\begin{gathered}
\varepsilon a(s) \phi^{\prime}(s, \varepsilon)=\left\{b(s)-\varepsilon a^{\prime}(s)\right\} \phi(s, \varepsilon), \\
{\left[\phi(s, \varepsilon) a(s) \exp \left(\frac{x s}{\varepsilon}\right)\right]_{\gamma}=0,}
\end{gathered}
$$

where

$$
a(s)=p_{11} s^{2}+p_{21} s+p_{31}, \quad b(s)=s^{3}+p_{10} s^{2}+p_{20} s+p_{30} .
$$

The equation (3.3) has a unique solution (up to a constant factor), which can be written in the form

$$
\phi(s, \varepsilon)=\frac{1}{a(s)} \exp \left(\frac{1}{\varepsilon} \int^{s} \frac{b(t)}{a(t)} d t\right) .
$$

Then $y(x, \varepsilon)$ becomes

$$
y(x, \varepsilon)=\int_{r} \frac{1}{a(s)} \exp \frac{1}{\varepsilon}\left(x s+\int^{s} \frac{b(t)}{a(t)} d t\right) d s
$$

and the contour $\gamma$ must satisfy (3.4), that is

$$
\left[\exp \left(\frac{1}{\varepsilon} S(s, x)\right)\right]_{\gamma}=0, \quad S(s, x)=x s+\int^{s} \frac{b(t)}{a(t)} d t .
$$

Solutions $s=s(x)$ of the equation

$$
\frac{\partial}{\partial s} S(s, x)=x+\frac{b(s)}{a(s)}=0
$$

or

$$
\begin{aligned}
k(s, x) & =b(s)+x a(s) \\
& =s^{3}+\left(p_{10}+x p_{11}\right) s^{2}+\left(p_{20}+x p_{21}\right) s+p_{30}+x p_{31}=0
\end{aligned}
$$

are called saddle points of (3.7) or $S(s, x)$. The zeros of $a(s)$ and the saddle points play important roles in the derivation of asymptotic expansions of (3.7). 
We note that the saddle points of the integral (3.7) are just the characteristic roots $\lambda(x)$ of the equation (3.1) which depend on $x$, and then the turning points are those where at least two saddle points coalesce.

Now we classify the equation (3.1) by means of zeros of $a(s)$, and indicate a general principle for choosing the contours $\gamma$ which we call admissible contours.

Case I. $\quad a(s)=p_{11}\left(s-a_{1}\right)\left(s-a_{2}\right) \quad\left(p_{11} \neq 0, a_{1} \neq a_{2}\right)$.

In this case, $\phi(s, \varepsilon)$ has a form

$$
\phi(s, \varepsilon)=\frac{1}{p_{11}}\left(s-a_{1}\right)^{\mu_{1} / \varepsilon-1}\left(s-a_{2}\right)^{\mu_{2} / \varepsilon-1} \exp \left(\frac{1}{\varepsilon} R(s)\right),
$$

where $R(s)$ is a polynomial of degree 2 , and $\mu_{j}=\lim _{s \rightarrow a_{j}}\left(s-a_{j}\right)(b(s) / a(s))$.

Since $R(s)$ has degree 2, the condition (3.8) is satisfied as $s \rightarrow \infty$ in appropriate two sectors. Choosing one of those sectors, an admissible contour $\gamma$ is obtained by taking a curve coming from infinity in the chosen sector and going out to infinity in another of the sectors where the condition (3.8) is satisfied.

Next, if $\mu_{1}$ or $\mu_{2}$ is zero then by taking as an admissible contour $\gamma$ a small circle enclosing $a_{1}$ or $a_{2}$ only we can obtain an exact solution of (3.1) by applying the residue theorem to the function (3.7).

When $\mu_{j}(j=1,2)$ are not zero, we form an admissible contour $\gamma$ consisting of three parts:

(1) Any curve coming from infinity in a sector where (3.8) is satisfied and not passing through $a_{k}(k \neq j)$ to the point of intersection with the circle $\left|s-a_{j}\right|$ $=\rho, \rho$ sufficiently small,

(2) One complete counter clockwise circuit of the circle $\left|s-a_{j}\right|=\rho$.

(3) Part (1) traversed in the opposite direction (Fig. 3.1).

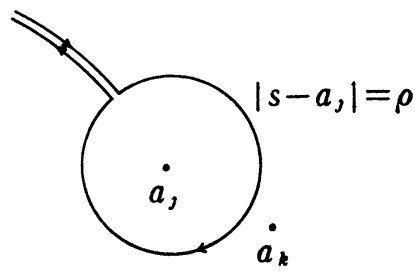

Fig. 3.1 The admissible contouy $\gamma$ for Case I.

By these construction of admissible contours $\gamma$ we can define several solutions of (3.1).

Case II.

$$
a(s)=p_{11}(s-a)^{2} \quad\left(p_{11} \neq 0\right) .
$$


In this case, $\phi(s, \varepsilon)$ has a form

$$
\phi(s, \varepsilon)=\frac{1}{p_{11}}(s-a)^{\mu / \varepsilon-2} \exp \frac{1}{\varepsilon}\left(\frac{\mu^{\prime}}{s-a}+R(s)\right),
$$

where $\mu$ and $\mu^{\prime}$ are constant and $R(s)$ is the same as in the case I.

As admissible contours $\gamma$ we can take the same curves as those defined in the case I. Moreover, since there is a half plane with a vertex $a$ where the function $\phi(s, \varepsilon)$ tends to zero as $s \rightarrow a$, we form contours as curves starting from $a$ in the half plane and join it to infinity or to itself after a complete circuit around $a$.

Case III. When $a(s)$ is a linear function or a non-zero constant, all possible admissible contours can be obtained as one of the contours defined in the case I.

The integrals with respect to these admissible contours $\gamma$ converge for all $x$, and it may be possible to obtain asymptotic expansions of these integrals by using the saddle point method. We compare the asymptotic expansions thus obtained with the formal WKB solutions (2.10) defined in the section 2 .

Here let us apply formally the saddle point method to the function $y(x, \varepsilon)$ of (3.7). We assume that the admissible contour $\gamma$ can be deformed without changing the value of $y(x, \varepsilon)$ so that it passes through a saddle point $\lambda(x)$ in an appropriate direction.

Let $x$ be not a turning point, then $\lambda(x)$ is a simple saddle point. Then the saddle point method gives us

$$
y(x, \varepsilon) \sim \sqrt{\frac{-2 \pi \varepsilon}{S^{\prime \prime}(\lambda(x))}}\left[\frac{1}{a(\lambda(x))}+O(\varepsilon)\right] \exp \frac{1}{\varepsilon}(S(\lambda(x))) \quad(\varepsilon \rightarrow 0),
$$

where

$$
S(\lambda(x))=S(\lambda(x), x)=\left[x s+\int^{s} \frac{b(t)}{a(t)} d t\right]_{s=\lambda(x)}
$$

and

$$
S^{\prime \prime}(\lambda(x))=\frac{\partial^{2}}{\partial s^{2}} S(\lambda(x), x)
$$

The choice of a branch of the root is as follows: arg $\sqrt{-S^{\prime \prime}(\lambda(x))}$ is equal to the angle between the positive direction of the tangent to the line of steepest descent $\gamma$ passing through $\lambda(x)$ and the positive direction of the real axis.

Since $s=\lambda(x)$ is a simple saddle point, then a small neighborhood of $s=$ $\lambda(x)$ in the complex $s$-plane is divided into four sectors by the curves $\operatorname{Re} S(s, x)$ $=\operatorname{Re} S(\lambda(x))$. Two sectors in which $\operatorname{Re} S(s, x)<\operatorname{Re} S(\lambda(x))$ are called valley regions and in the other two sectors we have $\operatorname{Re} S(s, x)>\operatorname{Re} S(\lambda(x))$ and we call them mountain regions. We introduce a definition of the $\lambda(x)$-saddle contour as a curve passing through $\lambda(x)$ which comes up from a vally region and goes down to another valley region (Fig. 5.2). 
Let $X$ be the Riemann surface of the algebraic function $\lambda(x)$ defined by $k(\lambda, x)=0$, and we define a notion of the admissible domain $\mathscr{D}$ of $\lambda(x)$-saddle contour. $\mathscr{D}$ is a simply connected and unbounded domain whose image under the mapping $s=\lambda(x)$ becomes a simply connected and unbounded domain in the complex s-plane, which we denote by $\mathscr{G}=\lambda(\mathscr{D})$, and satisfies the following conditions :

(i) For all $x \in \mathscr{D}$, there exists an admissible contour $\gamma(x)$ in $\mathcal{G}$ which passes through a unique saddle point $\lambda(x)$.

(ii) For all points $s=\lambda(\tilde{x})$ on the contour $\gamma(x)$, the contour $\gamma(x)$ is a $\lambda(\tilde{x})$ saddle contour passing through $s=\lambda(\tilde{x})$.

(iii) $\mathscr{D}$ is maximal. This means that if $x$ is continued to the outside of $\mathscr{D}$ it is impossible to draw any admissible and $\lambda(x)$-saddle contour, which satisfies the above condition (i) and (ii), without changing value of the integral.

We remark here that the admissible domain $\mathscr{D}$ of $\lambda(x)$-saddle contour is not unique for a characterestic root $\lambda(x)$.

Suppose that $x$ is in $\mathscr{D}_{j}$ which is an admissible domain of $\lambda_{j}(x)$-saddle contour. Then there exists an admissible and $\lambda_{j}(x)$-saddle contour $\gamma_{j}(x)$ in the $s$ plane which passes through $s=\lambda_{j}(x)$, and accordingly there exists an asymptotic solution $y_{j}(x, \varepsilon)$ of the form (3.11). The asymptotic expansion (3.11) is valid for $x$ as long as the above path $\gamma_{j}(x)$ is possible to be drawn without passing through other saddle points $\lambda_{k}(x)(k \neq j)$. On the other hand, the bounding curves of valley regions at the point $\lambda_{j}(x)$ is $\operatorname{Re} S(s, x)=\operatorname{Re} S\left(\lambda_{j}(x)\right)$. Therefore the boundary curves of $\mathscr{D}$, are the locus of points on which

$$
\operatorname{Re} S\left(\lambda_{j}(x)\right)=\operatorname{Re} S\left(\lambda_{k}(x)\right) \quad(j \neq k),
$$

and emerges from the turning point $x_{0}$ with $\lambda_{j}\left(x_{0}\right)=\lambda_{k}\left(x_{0}\right)$.

Since we have

$$
\frac{d}{d x} S\left(\lambda_{j}(x)\right)=\lambda_{j}(x)
$$

then these curves are just the Stokes curves defined in the section 1:

$$
\begin{aligned}
\operatorname{Re} \xi_{j k}\left(x_{0}, x\right) & =\operatorname{Re} \int_{x_{0}}^{x}\left\{\lambda_{j}(t)-\lambda_{k}(t)\right\} d t \\
& =\operatorname{Re} S\left(\lambda_{j}(x)\right)-\operatorname{Re} S\left(\lambda_{k}(x)\right)=0
\end{aligned}
$$

or defined by a differential equation

$$
\frac{d x_{R}}{d x_{I}}=\frac{\operatorname{Im}\left[\lambda_{j}(x)-\lambda_{k}(x)\right]}{\operatorname{Re}\left[\lambda_{j}(x)-\lambda_{k}(x)\right]}
$$

where $x=x_{R}+i x$.

At present we can not say further about general properties of (1) the admissible domain of $\lambda(x)$-saddle contour and (2) analytic continuations of the solution $y_{j}(x, \varepsilon)$, but we will discuss these problems (1) and (2) for individual 
equations as examples in the later sections.

\section{§4. Example I}

Firstly we consider the following equation :

$$
\varepsilon^{3} y^{\prime \prime \prime}-\varepsilon^{2} y^{\prime \prime}-\varepsilon x y^{\prime}+x y=0 .
$$

The characteristic equation is

$$
k(\lambda, x)=\lambda^{3}-\lambda^{2}-x \lambda+x=0
$$

and the characteristic roots are

$$
\lambda_{1}(x)=1, \quad \lambda_{2}(x)=\sqrt{x}, \quad \lambda_{3}(x)=-\sqrt{x} .
$$

The point $x=0$ is a simple turning point, but $x=1$ is not a simple turning point since the condition (2.21) is violated.

The equations of Stokes curves are given by

$$
\operatorname{Re} \xi_{21}(1, x) \equiv \operatorname{Re} \int_{1}^{x}(\sqrt{x}-1) d x=\operatorname{Re}\left(\frac{2}{3} x^{3 / 2}-x+\frac{1}{3}\right)=0
$$

and

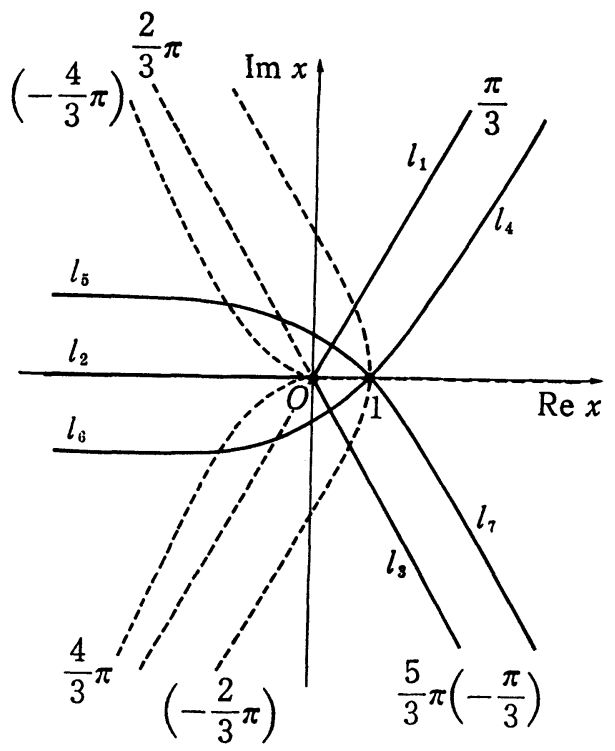

Fig. 4.1. Stokes curves (solid lines) $\operatorname{Re} \xi_{21}(1, x)=0, \operatorname{Re} \xi_{23}(0, x)$ $=0$, and anti Stokes curves (broken lines) $\operatorname{Im} \xi_{21}(1, x)=0, \operatorname{Im} \xi_{23}(0, x)$ $=0$ for (4.1). These curves are on two sheets of complex plane. 


$$
\operatorname{Re} \xi_{23}(0, x) \equiv 2 \operatorname{Re} \int_{0}^{x} \sqrt{x} d x=\operatorname{Re} \frac{4}{3} x^{3 / 2}=0 .
$$

The Stokes curve configuration is illustrated in Fig. 4.1. There are three Stokes curves issuing from $x=0$, which we label $l_{1}, l_{2}$ and $l_{3}$, and four Stokes curves from $x=1$, which we label $l_{4}, l_{5}, l_{6}$ and $l_{7}$. The level curves $\operatorname{Re} \xi_{21}(1, x)=$ const., $\operatorname{Im} \xi_{21}(0, x)=$ const. and $\operatorname{Re} \xi_{23}(0, x)=$ const., $\operatorname{Im} \xi_{23}(0, x)=$ const. are given in the Fig. 4.2(a) and Fig. 4.2(b) respectively, when the branch cut is given by $l_{3}$. Thus the equation (4.1) has an exact solution of the form

$$
y_{1}(x, \varepsilon)=A_{1} \exp \frac{x}{\varepsilon}
$$

and two formal WKB solutions of the form

$$
\tilde{y}_{2}(x, \varepsilon)=A_{2} x^{-1 / 4}(\sqrt{x}-1)^{-1} \exp \frac{2}{3 \varepsilon} x^{3 / 2},
$$

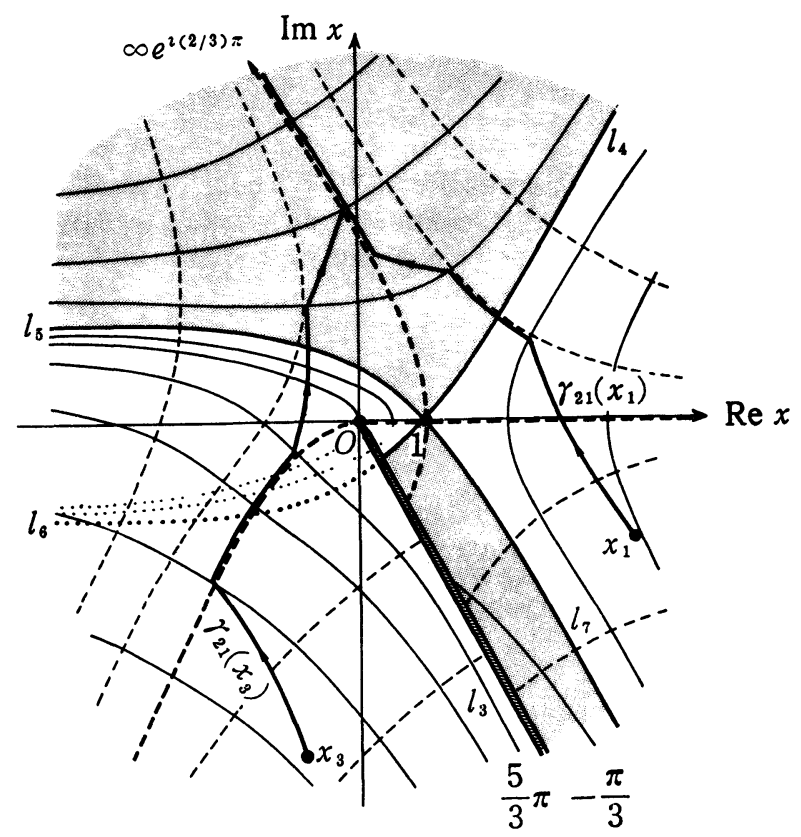

Fig. 4.2(a). Level curves of $\operatorname{Re} \xi_{21}(1, x)=$ const. (solid and dotted), $\operatorname{Im} \xi_{21}(1, x)=$ const. (broken) and a $(2,1)$-canonical path $\gamma_{21}\left(x_{\jmath}\right)\left(x_{j} \in S_{\jmath}\right)$ (cf. Fig. 4.4(a)). $l_{3}$ is the branch cut. Dotted lines are on the second sheet. In the shadow zone $\operatorname{Re} \xi_{21}(1, x) \leqq 0$ and in the other zones $\operatorname{Re} \xi_{21}(1, x) \geqq 0$. 


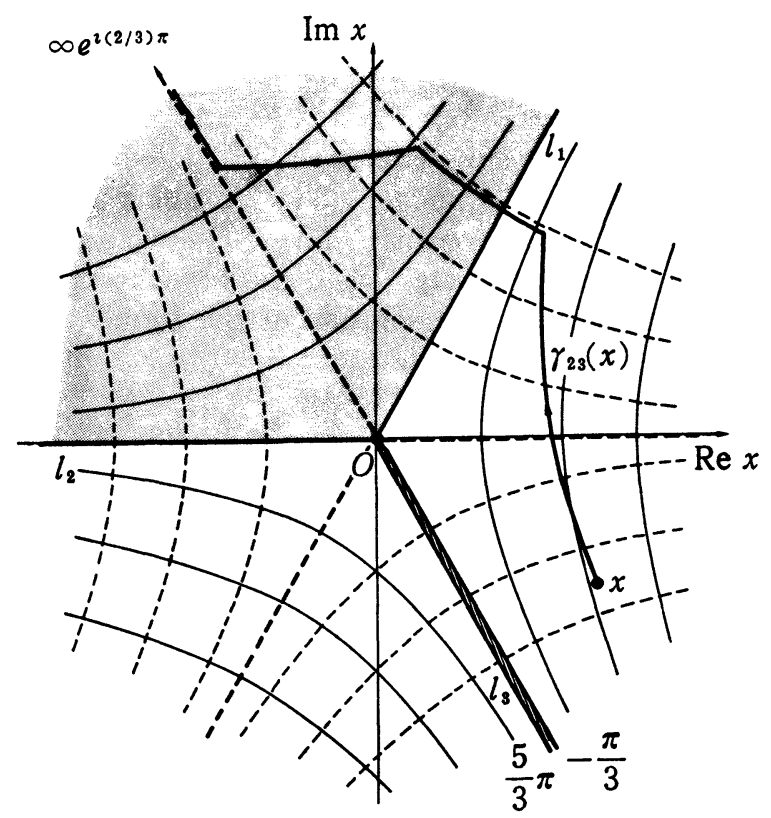

Fig. 4.2(b). Level curves $\operatorname{Re} \xi_{23}(0, x)=$ const. (solid), $\operatorname{Im} \xi_{23}(0, x)$ $=$ const. (broken) and a (2,3)-canonical path $\gamma_{23}(x)\left(x \in S_{1}\right) . \quad l_{3}$ is the branch cut. In the shadow zone $\operatorname{Re} \xi_{28}(0, x) \leqq 0$ and in the other zones $\operatorname{Re} \xi_{23}(0, x) \geqq 0$.

$$
\tilde{y}_{3}(x, \varepsilon)=A_{3} x^{-1 / 4}(\sqrt{x}+1)^{-1} \exp \left(-\frac{2}{3 \varepsilon} x^{3 / 2}\right) .
$$

Here and hereafter, a letter $A$ or $A$, means a constant number independent of $x$.

Now we show that corresponding to the characterestic roots $\pm \sqrt{x}$ there are $\sqrt{x}$-admissible domains $\mathscr{D}_{2}{ }^{(1)}$ and $\mathscr{D}_{2}{ }^{(2)}$, and $-\sqrt{x}$-admissible domain $\mathscr{D}_{3}$ which we illustrate as unshaded regions in Fig. 4.3(a), (b), (c).

From the definition, the $\sqrt{x}$-admissible domains consist of points from each of which we can draw a 2-canonical vector path $\Gamma_{2}(x)=\left\{\gamma_{21}(x), \gamma_{22}(x), \gamma_{23}(x)\right\}$.

The function of $t: \operatorname{Re} \xi_{21}(t, x)=\operatorname{Re} \int_{t}^{x}(\sqrt{ }-1) d s$ increases when $t$ moves from $x$ to $\infty$ on the $(2,1)$-canonical path $\gamma_{21}(x)$, and then the function of $t: \operatorname{Re} \xi_{21}(1, t)=\operatorname{Re} \int_{1}^{t}(\sqrt{s}-1) d s$ decreases $t$ moves from $x$ to $\infty$ along $\gamma_{21}(x)$.

Analogously the function of $t: \operatorname{Re} \xi_{23}(0, t)=\operatorname{Re} \int_{0}^{t} \sqrt{s} d s$ decreases when $t$ moves from $x$ to $\infty$ along the (2,3)-canonical path $\gamma_{23}(x)$.

We take the branch of $x^{3 / 2}$ such that $\operatorname{Re} x^{3 / 2}$ is negative in the sector $\pi / 3$ 
$<\arg x<\pi$, Let $\mathscr{D}_{21}$ and $\mathscr{D}_{23}$ be sets of points from which the two canonical paths $\gamma_{21}(x)$ and $\gamma_{23}(x)$ can be drawn respectively.

To construct the set $\mathscr{D}_{21}$, we define regions $S_{\imath}(i=1,2,3,4,5)$ and $S_{6}{ }^{\prime}$ as follows, see Fig. 4.4(a).

$S_{1}$ is a region bounded by $l_{7}$ and $l_{4}$ with $-\pi / 3<\arg x<\pi / 3$,

$S_{2}$ is a region bounded by $l_{4}$ and $l_{5}$ with $\pi / 3<\arg x<\pi$,

$S_{3}$ is a region bounded by $l_{6}$ and $l_{7}$ with $\pi<\arg x<5 \pi / 3$,

$S_{4}$ is a region bounded by $l_{2}, l_{5}$ and the segment $[0,1]$ with $0<\arg x<\pi$,

$S_{6}$ is a region bounded by $l_{2}, l_{6}$ and the segment $[0,1]$ with $\pi<\arg x<2 \pi$,

$S_{5}{ }^{\prime}$ is a region bounded by $l_{2}, l_{6}$ and the segment $[0,1]$ with $-\pi<\arg x<0$.

Now we consider a variation of the function of $t: \operatorname{Re} \xi_{21}(1, t)$ in the neighborhoods of the lines $l_{4}$ and $l_{2}$. Let the point $t$ on the curve $\gamma_{21}(x)$ be expressed as $t=r(s) \exp \{i \theta(s)\}$ by using a real parameter $s$.

Since we have

$$
\begin{aligned}
\frac{d}{d s} \operatorname{Re} \xi_{21}(1, t) & =\frac{d}{d s} \operatorname{Re}\left(\frac{2}{3} t^{3 / 2}-t+\frac{1}{3}\right) \\
& =\left(r^{1 / 2} \cos \frac{3}{2} \theta-\cos \theta\right) \frac{d r}{d s}-\left(r^{3 / 2} \sin \frac{3}{2} \theta-r \sin \theta\right) \frac{d \theta}{d s},
\end{aligned}
$$

the derivative $d\left\{\operatorname{Re} \xi_{21}(1, t)\right\} / d s<0$ by taking $d r / d s$ almost zero and $d \theta / d s$ positive (negative) for $\theta$ nearly $\pi / 3$ or $-\pi(\pi)$ and $r$ sufficiently large. By taking these facts into our consideration, we can draw $(2,1)$-canonical paths $\gamma_{21}(x)$ which connect $x$ and $\infty \exp (i 2 \pi / 3)$ and along which the function $\operatorname{Re} \xi_{21}(1, t)$

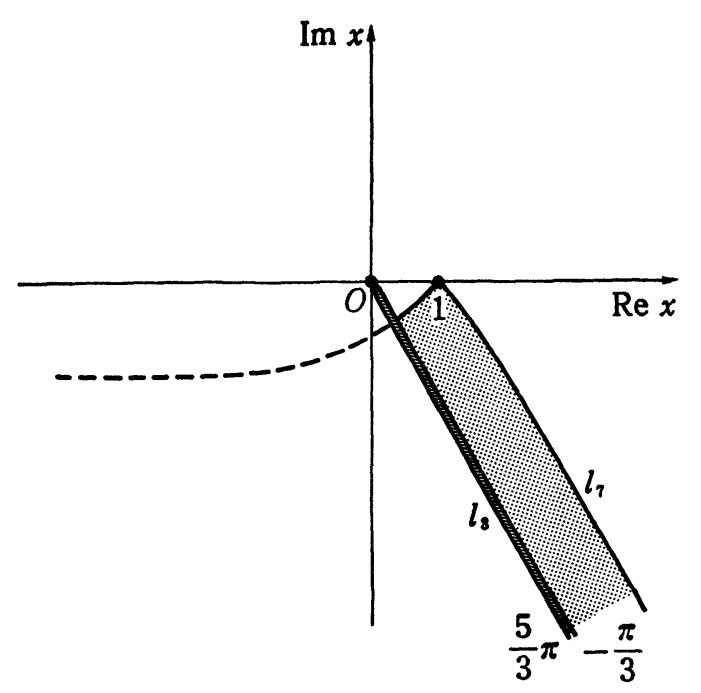

Fig. 4.3(a). The $\sqrt{x}$-admissible domain $\mathscr{D}_{2}{ }^{(1)}$ (unshaded), $l_{3}$ is the branch cut. 


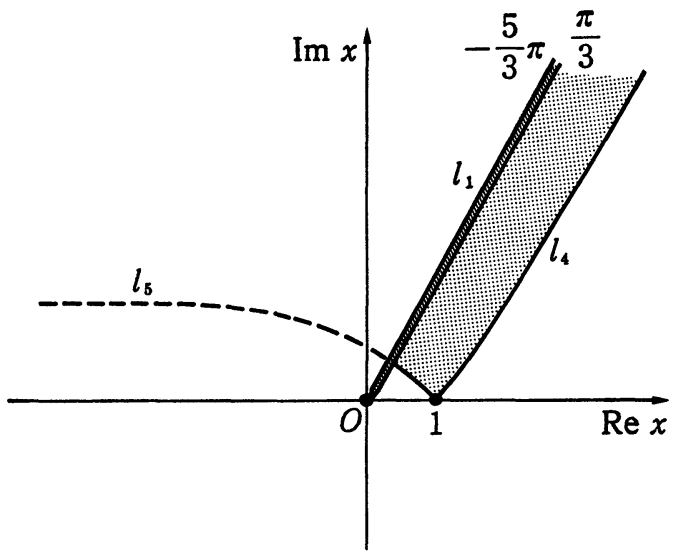

Fig. 4.3(b). The $\sqrt{x}$-admissible domain $\mathscr{D}_{2}{ }^{(2)}$ (unshaded), $l_{1}$ is the branch cut.

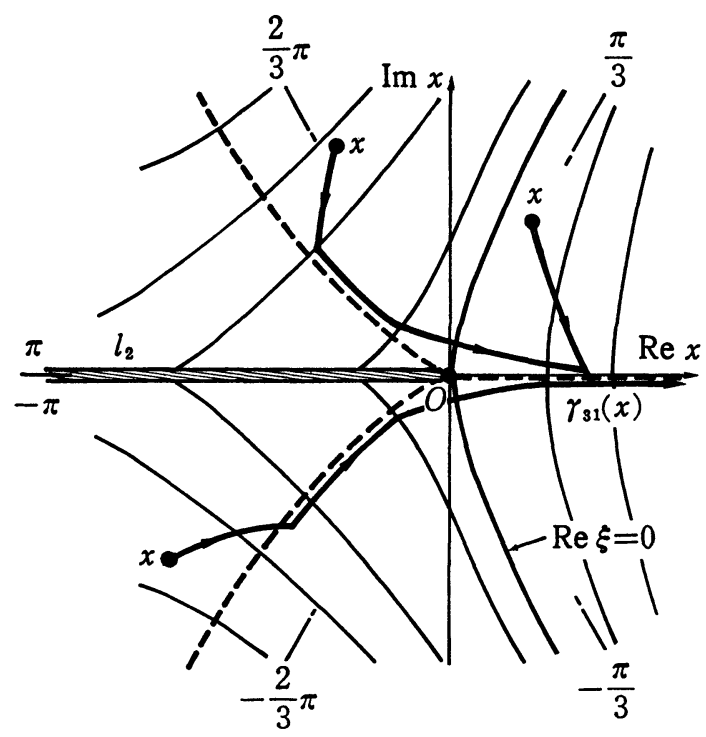

Fig. 4.3(c). The $-\sqrt{x}$-admissible domain $\mathscr{D}_{3}$ (unshaded) and $(3,1)$-canonical paths $\gamma_{31}(x) . \quad l_{2}$ is the branch cut. Solid and broken lines are level curves defined by $\operatorname{Re} \xi=$ const. and $\operatorname{Im} \xi=0$ $\left(\xi=\int_{0}^{x}\left(\lambda_{3}-\lambda_{1}\right) d x\right)$ respectively. Level curves are symmetric with respect to the real axis. 
decreases.

For typical points $x_{2}$ in $S_{\imath},(2.1)$-canonical paths $\gamma_{21}\left(x_{i}\right)$ are illustrated in Fig. 4.4(a). For example, for $x_{1} \in S_{1}$ the $(2,1)$-canonical path $\gamma_{21}\left(x_{1}\right)$ starts $x_{1}$, goes to a neighboring point of $l_{4}$ crossing the level curves $\operatorname{Re} \xi_{21}(1, t) \doteqdot$ $\operatorname{Re} \xi_{21}\left(1, x_{1}\right)=$ const. traverses $l_{4}$ into the region $S_{2}$ and then tends to $\infty \exp (i 2 \pi / 3)$ (Fig. 4.2(a)). Thus we can conclude that

$$
\mathscr{D}_{21}=S_{1} \cup l_{4} \cup S_{2} \cup l_{5} \cup S_{4} \cup l_{2} \cup S_{5} \cup l_{6} \cup S_{3} \cup S_{5}{ }^{\prime} \cup[0,1] \quad \text { (Fig. 4.4(a)). }
$$

Similarly it is easy to see that

$$
\mathscr{D}_{23}=\{x \mid-\pi / 3<\arg x<5 \pi / 3\}
$$

Since for any $x$ we can take a curve connecting $x$ and $\infty \exp (i 2 \pi / 3)$ as $\gamma_{22}(x)$, we have obtained a $\sqrt{x}$-admissible domain $\mathscr{D}_{2}^{(1)}$ as the intersection of $\mathscr{D}_{21}$ and $\mathscr{D}_{23}$, which is illustrated in Fig. 4.3(a).

Next, if the branch of $x^{3 / 2}$ is taken such that $\operatorname{Re} x^{3 / 2}$ is negative in the sector $-\pi<\arg x<-\pi / 3$, we have by the same way another $\sqrt{x}$-admissible domain $\mathscr{D}_{2}{ }^{(2)}$ as given by Fig. $4.3(\mathrm{~b})$.

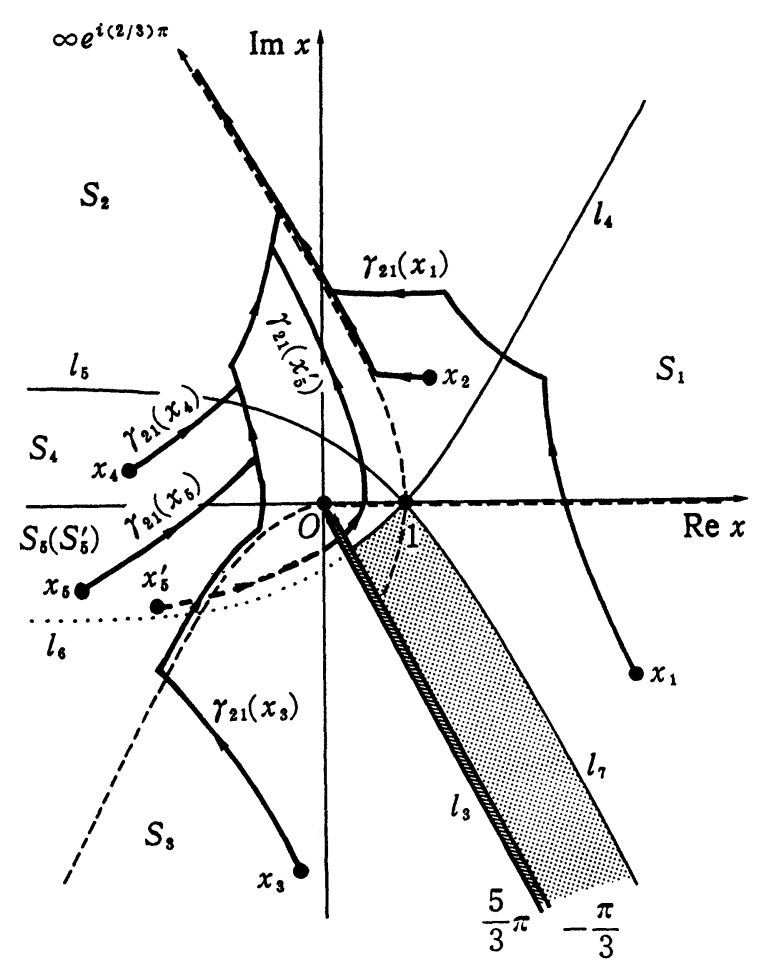

Fig. 4.4(a). $\mathscr{D}_{21}$ (unshaded) and $(2,1)$-canonical paths $\gamma_{21}\left(x_{j}\right), x_{j} \in S_{\jmath}$. (cf. Fig. 4.2(a)). 


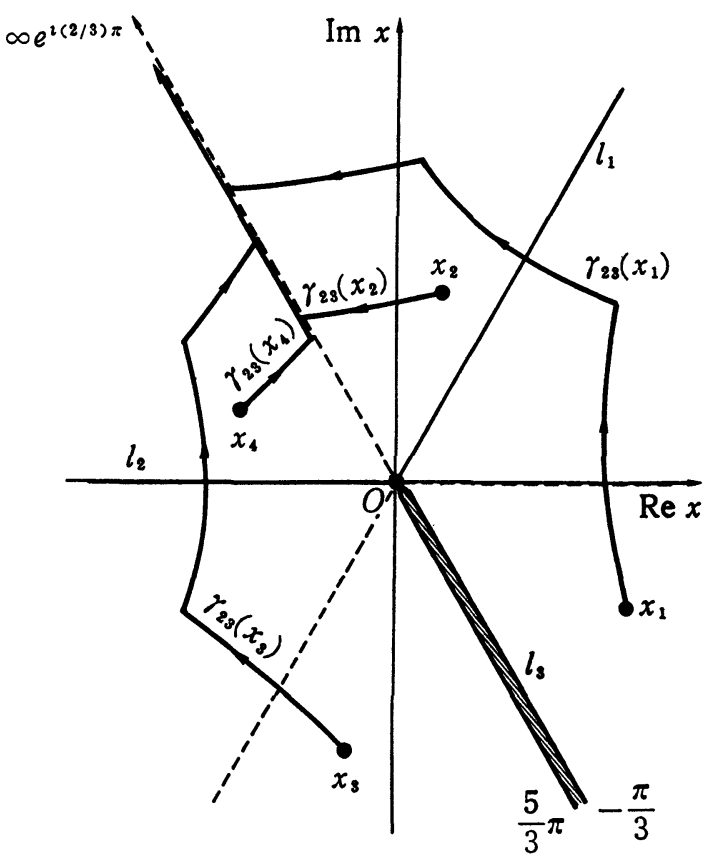

Fig. 4.4(b). $\mathscr{D}_{23}$ with the branch cut along $l_{3}$, and

$(2,3)$-canonical paths $\gamma_{23}(x)$. (cf. Fig. 4.2(b)).

Lastly we can define a $-\sqrt{x}$-admissible domain $\mathscr{D}_{3}$ for which $\operatorname{Re}\left(-x^{3 / 2}\right)$ is negative in the sector $-\pi / 3<\arg x<\pi / 3$ (Fig. 4.3(c)). Therefore we obtain the WKB solutions $y_{2}{ }^{(1)}(x, \varepsilon)$ and $y_{2}^{(2)}(x, \varepsilon)$ of (4.1) whose asymptotic expansions coincide with the formal WKB solution (4.3) in the domain $\mathscr{D}_{2}{ }^{(1)}$ and $\mathscr{D}_{2}{ }^{(2)}$ respectively, and the WKB solution $\tilde{y}_{3}(x, \varepsilon)$ with the asymptotic expansion (4.4) in $\mathscr{D}_{3}$.

On the other hand, from (3.7) and (3.8) solutions defined by the Laplace integral become in this case

$$
y(x, \varepsilon)=A \int_{\gamma} \frac{1}{s-1} \exp \frac{1}{\varepsilon}\left(x s-\frac{s^{3}}{3}\right) d s
$$

with a constant $A$ and a contour $\gamma$ satisfying

$$
\left[\exp \frac{1}{\varepsilon}\left(x s-\frac{s^{3}}{3}\right)\right]_{r}=0 \text {. }
$$

Corresponding to the pole $s=1$ of the integrand of (4.5) ( $\$ 3$, Care I), there is an exact solution $y_{1}(x, \varepsilon)$ of $(4.1)$ of the form 


$$
y_{1}(x, \varepsilon)=2 \pi i A \exp \left(-\frac{1}{3 \varepsilon}\right) \exp \left(\frac{x}{\varepsilon}\right) .
$$

This coincides with (4.2).

The saddle points of $S(s, x)=x s-s^{3} / 3$ are $\pm \sqrt{x}$, and we have

$$
S(s, x)= \pm \frac{2}{3} x^{3 / 2} \mp \sqrt{x}(s \mp \sqrt{x})^{2}-\frac{1}{3}(s \mp \sqrt{x})^{3} .
$$

In the followings, we define three admissible and $\pm \sqrt{x}$-saddle contours in the complex $s$-plane. Let $\mathcal{S}_{\imath}(i=1,2,3)$ be sectors defined by

$$
\begin{aligned}
& \mathcal{S}_{1}:-\frac{\pi}{6}<\arg s<\frac{\pi}{6}, \\
& \mathcal{S}_{2}: \quad \frac{\pi}{2}<\arg s<\frac{5 \pi}{6}, \\
& \mathcal{S}_{3}: \quad \frac{7 \pi}{6}<\arg s<\frac{3 \pi}{2} .
\end{aligned}
$$

Then the curve $\gamma_{2}^{(1)}$ is the admissible and $\sqrt{x}$-saddle contour which satisfies that it passes through the point $\sqrt{x}$ in appropriate direction, passes above the point $s=1$, and comes from infinity in $\mathcal{S}_{2}$ and goes away to infinity in $\mathcal{S}_{1}$. Similarly the curve $\gamma_{2}{ }^{(2)}$ passes through the point $\sqrt{x}$ passes under the point $s=1$, comes from infinity in $\mathcal{S}_{1}$ and goes to infinity in $\mathcal{S}_{3}$. Lastly the curve $\gamma_{3}$ is the admissible and $-\sqrt{ } x$-saddle contour which comes from infinity in $\mathcal{S}_{3}$, passes through the point $-\sqrt{ } x$ and goes to infinity in $\mathcal{S}_{2}$, see Fig. 4.5.

By these choices of the contours $\gamma_{2}{ }^{(1)}, \gamma_{2}{ }^{(2)}$ and $\gamma_{3}$ as $\gamma$ in the expression (4.5), we can define three solutions $y_{2}^{(1)}(x, \varepsilon), y_{2}{ }^{(2)}(x, \varepsilon)$ and $y_{3}(x, \varepsilon)$ respectively. These three solutions and $y_{1}(x, \varepsilon)$ of $(4.7)$ satisfy the following connection rule by the Cauchy's integral theorem.

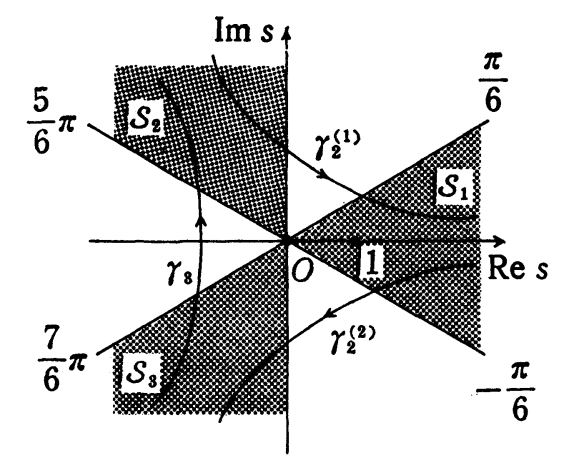

Fig. 4.5. The admissible and $\pm \sqrt{x}$-saddle contours. 


$$
y_{1}(x, \varepsilon)+y_{2}{ }^{(1)}(x, \varepsilon)+y_{2}{ }^{(2)}(x, \varepsilon)+y_{3}(x, \varepsilon)=0 .
$$

By applying the saddle point method, we have the asymptotic expansions

$$
\begin{aligned}
y_{2}^{(1)}(x, \varepsilon) & =A \int_{\gamma_{2}(1)} \frac{1}{s-1} \exp \left\{\frac{1}{\varepsilon}\left(x s-\frac{1}{3} s^{3}\right)\right\} d s \\
& \sim A \sqrt{\pi \varepsilon} \frac{x^{-1 / 4}}{\sqrt{x-1}} \exp \left(\frac{2}{3 \varepsilon} x^{3 / 2}\right) \quad(\varepsilon \rightarrow 0), \\
y_{2}^{(2)}(x, \varepsilon) & =A \int_{r_{2}(2)} \frac{1}{s-1} \exp \left\{\frac{1}{\varepsilon}\left(x s-\frac{1}{3} s^{3}\right)\right\} d s \\
& \sim-A \sqrt{\pi \varepsilon} \frac{x^{-1 / 4}}{\sqrt{x}-1} \exp \left(\frac{2}{3 \varepsilon} x^{3 / 2}\right) \quad(\varepsilon \rightarrow 0), \\
y_{3}(x, \varepsilon)= & A \int_{r_{3}} \frac{1}{s-1} \exp \left\{\frac{1}{\varepsilon}\left(x s-\frac{1}{3} s^{3}\right)\right\} d s \\
& \sim-A \sqrt{\pi \varepsilon i} \frac{x^{-1 / 4}}{\sqrt{x}+1} \exp \left(-\frac{2}{3 \varepsilon} x^{3 / 2}\right) \quad(\varepsilon \rightarrow 0) .
\end{aligned}
$$

Next, we consider the admissible domains of the $\pm \sqrt{x}$-saddle contour. It is possible to prove that these are just the same with the $\pm \sqrt{x}$-admissible domain $\mathscr{D}_{2}{ }^{(1)}, \mathscr{D}_{2}{ }^{(2)}$ and $\mathscr{D}_{3}$.

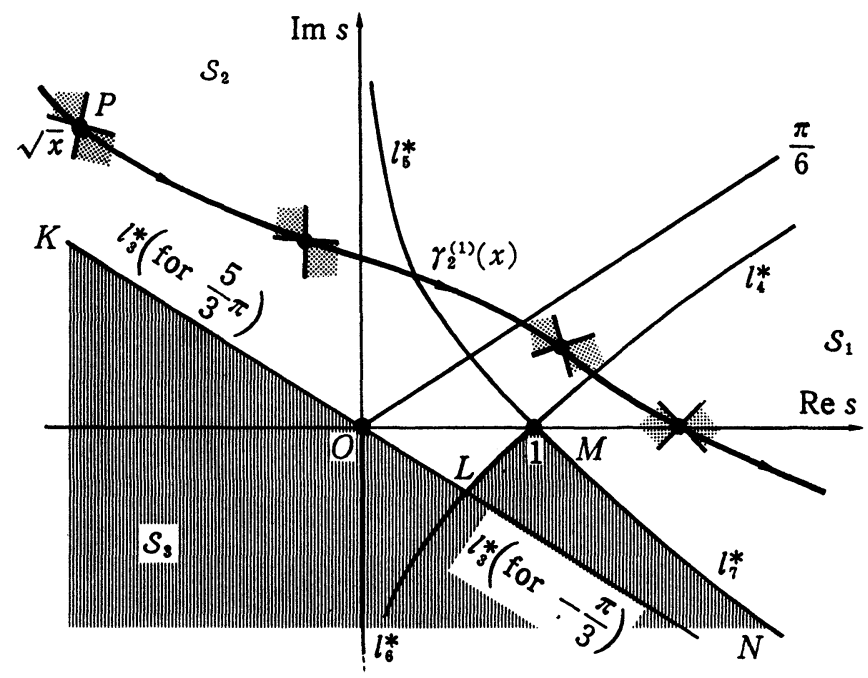

Fig. 4.6. $\mathcal{G}_{2}{ }^{(1)}$ (unshaded), $\sqrt{x}$-saddle contour $\gamma_{2}^{(1)}(x)$. Dotted parts are valley regions at each point. $l_{j}^{*}$ is the image of $l$, under the map $s=\sqrt{\bar{x}}, N=\infty e^{-(\pi / 6) i}, K=\infty e^{(6 / 6) \pi t}$.

For example, let in the $s$-plane $\mathcal{G}_{2}^{(1)}$ be the image of $\mathscr{D}_{2}^{(1)}$ in the $x$-plane under the mapping $s=\sqrt{x}$ (Fig. 4.6). From (4.8), the direction of the curve of 
steepest descent at the saddle point $\sqrt{x}$ is $\arg (s-\sqrt{x})=-(1 / 2) \arg \sqrt{x}$ and $(-1 / 2) \arg \sqrt{x} \pm \pi$. Then the valley regions at $\sqrt{x}$ are

$$
-\frac{1}{2} \arg \sqrt{x}-\frac{\pi}{4}<\arg (s-\sqrt{x})<-\frac{1}{2} \arg \sqrt{x}+\frac{\pi}{4}
$$

and

$$
-\frac{1}{2} \arg \sqrt{x}+\frac{3}{4} \pi<\arg (s-\sqrt{x})<-\frac{1}{2} \arg \sqrt{x}+\frac{5}{4} \pi .
$$

The $\sqrt{x}$-saddle contour must pass through the point $\sqrt{x}$ into the forme valley region (4.11). In Fig. 4.6, the velly regions (dotted) at $P$ are illustrated.

It is easy to see that for all $x$ in $\mathscr{D}_{2}{ }^{(1)}$ we can draw an admissible and $\sqrt{x}$-saddle contour $\gamma_{2}^{(2)}(x)$ in $\mathcal{G}_{2}{ }^{(1)}$, which satisfies that

(i) $\gamma_{2}^{(1)}(x)$ passes through a unique saddle point $\sqrt{ } \bar{x}$,

(ii) for all points $s=\sqrt{\tilde{x}}$ on the contour $\gamma_{2}^{(1)}(x)$ the contour $r_{2}^{(1)}(x)$ is a $\sqrt{x}$ saddle contour passing through $s=\sqrt{\tilde{x}}$, and

(iii) $\gamma_{2}{ }^{(1)}(x)$ passes above the point $M(s=1$, the singular point of the integrand), and extends to infinity in the sectors $\mathcal{S}_{1}$ and $\mathcal{S}_{2}$ (see Fig. 4.5 and 4.6).

Then to assert that $\mathscr{D}_{2}{ }^{(1)}$ is an admissible domain of the $\sqrt{x}$-saddle contour for $y_{2}{ }^{(1)}(x, \varepsilon)$, it is necessary to prove that $\mathscr{D}_{2}{ }^{(1)}$ is maximal. For the points below the line $K N$ (Fig. 4.6), the admissible and $\sqrt{x}$-saddle contour must extend to infinity in the sectors $\mathcal{S}_{2}$ and $\mathcal{S}_{3}$.

Next, the $\sqrt{x}$-saddle contours which pass through points below the curves $L M$ and $M N$ can not pass above the point $M$. Then the integrals defined by such contours does not define the function $y_{2}{ }^{(1)}(x, \varepsilon)$. Therefore we have proved that the admissible domain of $\sqrt{x}$-saddle contour for $y_{2}{ }^{(1)}(x, \varepsilon)$ is the $\sqrt{x}$ admissible domain $\mathscr{D}_{2}{ }^{(1)}$.

\section{§5. Example II}

We consider the following two differential equations :

$$
\begin{gathered}
\varepsilon^{3} y^{\prime \prime \prime}+i \varepsilon^{2} x y^{\prime \prime}+\varepsilon y^{\prime}+i x y=0, \\
\varepsilon^{3} y^{\prime \prime \prime}-\varepsilon^{2} x y^{\prime \prime}-\varepsilon y^{\prime}+x y=0 .
\end{gathered}
$$

The above two equations have essentially the same characteristic, but since each Stokes curve configuration or the admissible domains of $\lambda(x)$-saddle contour are seemed to be interesting we refer these equations as examples.

$1^{\circ}$ At first, we analyse (5.1) in detail. The characteristic equation for it becomes $k(\lambda, x)=\lambda^{3}+i x \lambda^{2}+\lambda+i x=\left(\lambda^{2}+1\right)(\lambda+i x)=0$, characteristic roots are $\pm i$, $-i x$, and then turning points are $x= \pm 1$. Both turning points are not simple, because the condition (2.21) does not hold.

The Stokes curve configuration is given in Fig. 5.1. 


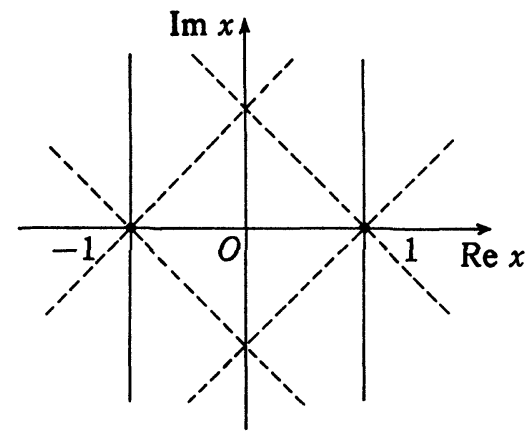

Fig. 5.1. Stokes curves (solid lines) and antiStokes curves (broken lines) for (5.1).

Solutions given by the Laplace integral have the form

$$
y(x, \varepsilon)=A \int_{\gamma} \frac{1}{s^{2}+1} \exp \frac{1}{\varepsilon}\left(-x s-\frac{i}{2} s^{2}\right) d s,
$$

where $A$ is constant. By putting $s=i t$, it becomes

$$
y(x, \varepsilon)=A \int_{r} \frac{i}{1-t^{2}} \exp \frac{i}{\varepsilon}\left(-x t+\frac{1}{2} t^{2}\right) d t .
$$

Taking as admissible contours $\gamma$ two small circles enclosing each of two poles $t= \pm 1$ of the integrand ( $\$ 3$, Case I), we obtain two exact solutions :

$$
\begin{aligned}
& y_{1}(x, \varepsilon)=A \pi \exp \left(\frac{i}{2 \varepsilon}-\frac{x i}{\varepsilon}\right), \\
& y_{2}(x, \varepsilon)=-A \pi \exp \left(\frac{i}{2 \varepsilon}+\frac{x i}{\varepsilon}\right) .
\end{aligned}
$$

Let us define $w(t, x)$ as

$$
w(t, x)=i\left(-x t+\frac{1}{2} t^{2}\right),
$$

then a saddle point of $w(t, x)$ is $x$, and

$$
w(t, x)=-\frac{i}{2} x^{2}+\frac{i}{2}(t-x)^{2} .
$$

Therefore (5.3) can be written

$$
y_{3}(x, \varepsilon)=A i \exp \left(-\frac{i}{2 \varepsilon} x^{2}\right) \int_{\gamma} \frac{1}{1-t^{2}} \exp \left(\frac{i}{2 \varepsilon}(t-x)^{2}\right) d t .
$$

Here we put 


$$
v(x, \varepsilon)=\int_{r} \frac{1}{1-t^{2}} \exp \left(\frac{i}{2 \varepsilon}(t-x)^{2}\right) d t .
$$

The asymptotic expansions of $v(x, \varepsilon)$ as $\varepsilon \rightarrow 0$ are obtained by applying the saddle point method except for neighborhoods of turning points. In this case, an admissible and $x$-saddle contour $\gamma(x)$ in the $t$-plane is as follows:

(1) $\gamma(x)$ comes from infinity in the third quadrant and extends to infinity in the first quadrant of the $t$-plane.

(2) at each $x, \gamma(x)$ passes through $x$ from the lower valley region to the upper one (see Fig. 5.2).

By this choice of contour, we have

$$
v(x, \varepsilon) \sim \frac{\sqrt{2 \pi \varepsilon}}{1-x^{2}} \exp \frac{\pi i}{4} \quad(\varepsilon \rightarrow 0),
$$

then the asymptotic expansion of $y_{3}(x, \varepsilon)$ becomes

$$
y_{3}(x, \varepsilon) \sim \sqrt{2 \pi \varepsilon} \exp \left(\frac{3 \pi i}{4}\right) \cdot \frac{1}{1-x^{2}} \exp \left(-\frac{i x^{2}}{2 \varepsilon}\right) \quad(\varepsilon \rightarrow 0)
$$

In this example, it is easy to get the admissible domains of a $x$-saddle contour and connection rules.

Suppose initially $x$ is in the first quadrant of the $t$-plane, then we can obtain the asymptotic expansion (5.5). This asymptotic expansion is valid as long as $y_{3}(x, \varepsilon)$ is analytically continued so that the admissible and $x$-saddle contour $\gamma(x)$ passing through $x$ is possible to be drawn without meeting turning points and singular points of the integrand of $y_{3}(x, \varepsilon)$. By this condition, there are three admissible domains of $x$-saddle contour, which we label $\mathscr{D}^{(1)}, \mathscr{D}^{(2)}$ and $\mathscr{D}^{(3)}$ as in Fig. 5.3.

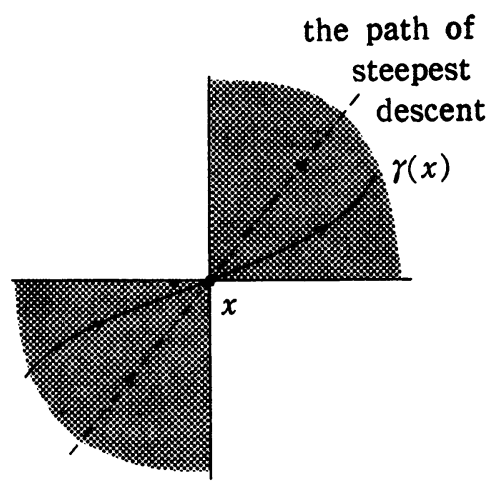

Fig. 5.2. Valley regions (shaded) in the neighbourhood of $x$. 


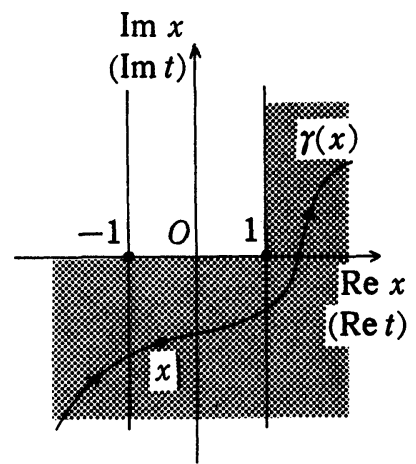

(a) $\mathscr{D}_{3}^{(1)}$

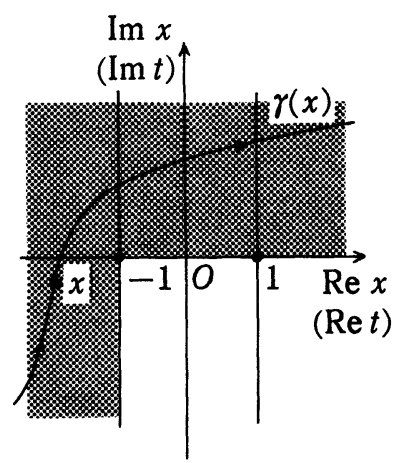

(b) $\mathscr{D}_{3}^{(2)}$

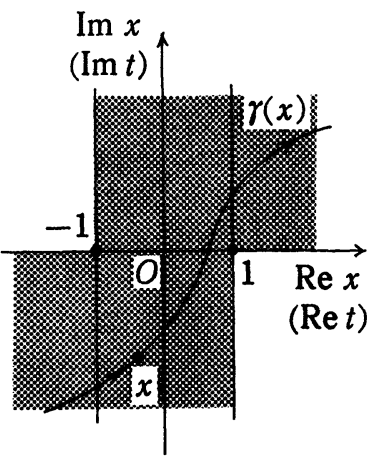

(c) $\mathscr{D}_{3}^{(3)}$

Fig. 5.3. Admissible domains $\mathscr{D}_{3}{ }^{(1)}$ (shaded) of $x$-saddle contour, in the $x$-plane, $\gamma(x)$ is the admissible contour passing through a saddle point $x$ in the $t$-plane.

To each domain $\mathscr{D}^{(i)}$, there corresponds the solution $y_{3}{ }^{(i)}(x, \varepsilon)(i=1,2,3)$. The following relations between $y_{1}(x, \varepsilon), y_{2}(x, \varepsilon)$ and $y_{3}{ }^{(i)}(x, \varepsilon)(i=1,2,3)$ are apparent from the Cauchys' intergral theorem

$$
\begin{aligned}
& y_{3}{ }^{(1)}-y_{3}{ }^{(2)}=y_{1}+y_{2}, \\
& y_{3}{ }^{(1)}-y_{3}{ }^{(3)}=y_{1}, \\
& y_{3}{ }^{(2)}-y_{3}{ }^{(2)}=-y_{2} .
\end{aligned}
$$

$2^{\circ}$ By analogous considerration, we can analyze the equation (5.2). The characteristic polynomial is

$$
k(\lambda, x)=\lambda^{3}-x \lambda^{2}-\lambda+x=\left(\lambda^{2}-1\right)(\lambda-x) .
$$

Turning points are $x= \pm 1$. The Stokes curve configuration is given in Fig. 5.4.

Solutions can be expressed by the integral

$$
y(x, \varepsilon)=A \int_{r} \frac{1}{t^{2}-1} \exp \frac{1}{\varepsilon}\left(x t-\frac{1}{2} t^{2}\right) d t .
$$

As before the residue theorem gives us two exact solutions:

$$
\begin{aligned}
& y_{1}(x, \varepsilon)=A \pi i \exp \left(-\frac{1}{2 \varepsilon}+\frac{x}{\varepsilon}\right), \\
& y_{2}(x, \varepsilon)=A \pi i \exp \left(-\frac{1}{2 \varepsilon}-\frac{x}{\varepsilon}\right) .
\end{aligned}
$$

By applying the saddle point method to (5.7), we can define four solutions $y_{3}{ }^{(i)}(x, \varepsilon)$, whose asymptotic expansions are of the form 


$$
y_{3}{ }^{(i)}(x, \varepsilon) \sim A \sqrt{2 \pi \varepsilon} \frac{1}{x^{2}-1} \exp \frac{x^{2}}{2 \varepsilon} \quad(\varepsilon \rightarrow 0 ; i=1,2,3,4) .
$$

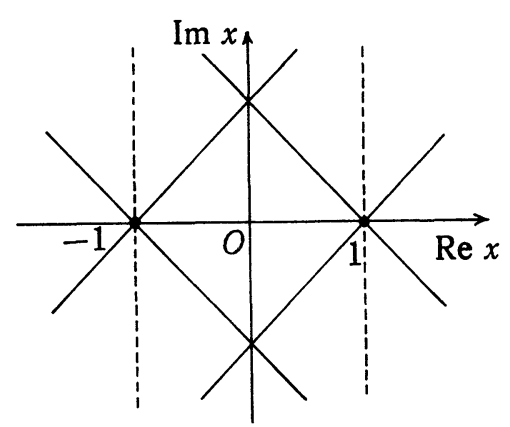

Fig. 5.4. Stokes curves (solid lines) and antiStokes curves (broken lines) for (5.2).

The admissible domain $\mathscr{D}^{(i)}$ of $x$-saddle contour for each $y_{3}{ }^{(i)}(x, \varepsilon)$ is illustrated in Fig. 5.5, and the following linear relations hold

$$
\begin{aligned}
& y_{3}{ }^{(1)}-y_{3}{ }^{(2)}=y_{1}+y_{2}, \\
& y_{3}{ }^{(1)}-y_{3}{ }^{(3)}=y_{1}, \\
& y_{3}{ }^{(1)}-y_{3}{ }^{(4)}=y_{2}, \\
& y_{3}{ }^{(4)}-y_{3}{ }^{(2)}=y_{1}, \\
& y_{3}{ }^{(3)}-y_{3}{ }^{(2)}=y_{2} .
\end{aligned}
$$

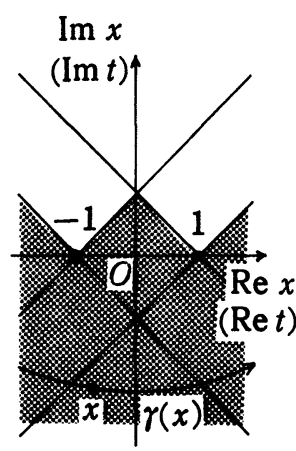

(a) $\mathscr{D}_{3}^{(1)}$

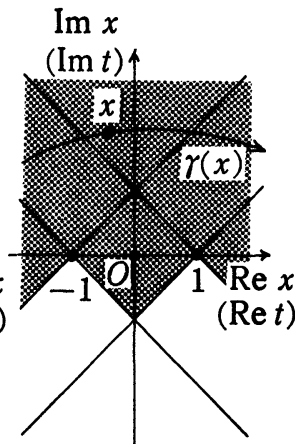

(b) $\mathscr{D}_{3}^{(2)}$

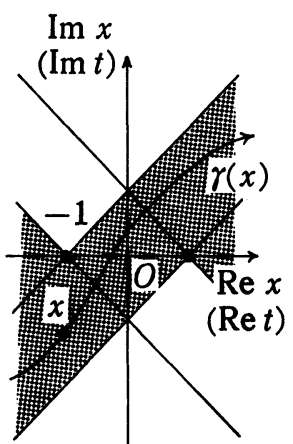

(c) $\mathscr{D}_{3}^{(3)}$

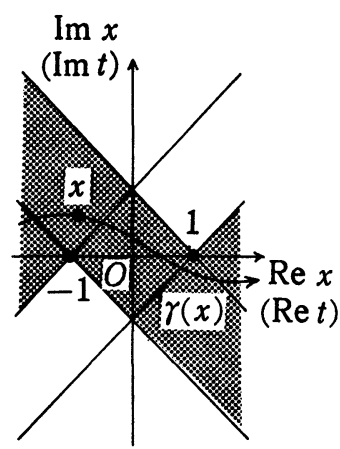

(d) $\mathscr{D}_{3}^{(4)}$

Fig. 5.5. Admissible domains $\mathscr{D}_{3}{ }^{(i)}$ (shaded) of $x$-saddle contour in the $x$-plane, $\gamma(x)$ is the admissible contour passing through a saddle point $x$ in the $t$-plane. 


\section{§6. Further examples}

In the sections 4 and 5 we analized rather simple three examples from the two aspects: the one is the theory of differential equations containing a small parameter, and the other is application of the saddle point method to the functions represented by the Laplace integral.

We remark here that in these examples there is no need to consider the secondary Stokes curves since the so-called Stokes phenomenon occurs only along the usual Stokes curves. Then we can say that, even in the cases where there exist multiple turning points and two turning points are joined by a Stokes curve, it is not always necessary to consider the secondary Stokes curves. These cases are excluded in the article [1].

To understand what new phenomena, such as the appearence of secondary Stokes curves, occur in the WKB theory of higher ordinary differential equations, it is desirable to analyze further examples that exhibit various features in the distribution of turning points or the Stokes curve configurations. Therefore in this section, we list up several examples of third order ordinary differential equations with linear coefficients which seem to be interesting.

The discriminant of the characteristic equation (2.2) is given by $q^{2}+4 p^{3}$, which is a 4 th degree polynomial for linear coefficients $p_{j}(x)(j=1,2,3)$. From the discriminant we see that the differential equation (1.1) possesses at most four turning points. The simplest equation is $\varepsilon^{3} y^{\prime \prime \prime}+x y=0$. This is called Turrittin type, and we excluded it here.

Case 1.

$$
\varepsilon^{3} \frac{d^{3} y}{d x^{3}}+3 \varepsilon \frac{d y}{d x}-i x y=0
$$

The characteristic equation is

$$
\lambda^{3}+3 \lambda-i x=0 .
$$

There are two simple turning points $x= \pm 2$, and the Laplace integral representation becomes

$$
y(x, \varepsilon)=A \int_{\gamma} \exp \frac{1}{\varepsilon}\left\{x s+i\left(\frac{s^{4}}{4}+\frac{3}{2} s^{2}\right)\right\} d s .
$$

This integral is a variant of the so-called Pearcey integral:

$$
F(\varepsilon, x, y)=\int_{-\infty}^{+\infty} f(s) \exp \frac{i}{\varepsilon}\left(s^{4}+x s^{2}+y s\right) d s .
$$

Case 2.

$$
\varepsilon^{3} \frac{d^{3} y}{d x^{3}}-3 \varepsilon x \frac{d y}{d x}+2 y=0
$$


The characteristic equation is

$$
\lambda^{3}-3 x \lambda+2=0 .
$$

There are three simple turning points $x=1, \omega, \omega^{2}$.

The integral representation becomes

$$
y(x, \varepsilon)=A \int_{\gamma} s^{1+2 / 3 \varepsilon} \exp \frac{1}{\varepsilon}\left(x s-\frac{s^{3}}{9}\right) d s .
$$

Case 3.

$$
\varepsilon^{3} \frac{d^{3} y}{d x^{3}}+3 \varepsilon^{2} x \frac{d^{2} y}{d x^{2}}+(x+1) y=0 .
$$

The characteristic equation is

$$
\lambda^{3}+3 x \lambda^{2}+(x+1)=0 .
$$

There are four simple turning points $x=-1,-\frac{1}{2}, \frac{1 \pm i \sqrt{7}}{4}$.

The integral representation becomes

$$
y(x, \varepsilon)=A \int_{\tau} \frac{1}{\left(3 s^{2}+1\right)^{1+1 / 13 \varepsilon}} \exp \frac{1}{\varepsilon}\left(x s+\frac{s^{2}}{6}+\frac{1}{\sqrt{3}} \tan ^{-1} \sqrt{3} s\right) d s .
$$

Case 4.

$$
\varepsilon^{3} \frac{d^{3} y}{d x^{3}}-3 \varepsilon^{2} x \frac{d^{2} y}{d x^{2}}+x y=0 .
$$

The characteristic equation is

$$
\lambda^{3}-3 x \hat{\lambda}^{2}+x=0 .
$$

There are a double turning point $x=0$ and two simple turning points $x= \pm 1 / 2$.

The integral representation becomes

$$
y(x, \varepsilon)=A \int_{r} \frac{1}{\left(3 s^{2}-1\right)^{1+1 / 18 \varepsilon}} \exp \frac{1}{\varepsilon}\left(x s-\frac{s^{2}}{6}\right) d s .
$$

Case 5.

$$
\varepsilon^{3} \frac{d^{3} y}{d x^{3}}+3 \varepsilon^{2} x \frac{d^{2} y}{d x^{2}}+3 \varepsilon x \frac{d y}{d x}+y=0 .
$$

The characteristic equation is

$$
\lambda^{3}+3 x \lambda^{2}+3 x \lambda+1=0 .
$$

There are a triple turning point $x=1$ and a simple turning point $x=-1 / 3$.

The integral representation becomes

$$
y(x, \varepsilon)=A \int_{r}(s+1)^{-1} s^{-1+1 / 3 \varepsilon} \cdot \exp \frac{1}{\varepsilon}\left(x s-\frac{s}{3}+\frac{s^{2}}{6}\right) d s .
$$


Aknowledgement: The authors are deeply indebted to the referee for his careful reading, many corrections and valuable suggestions.

\section{REFERENCES}

[1] Berk, W. L., W. M. Nevines and K.V. Roberts, New Stokes lines in WKB theory, J. Math. Phys. 23 (1982), pp. 988-1002.

[2] Evgrafov, M. A. AND M. B. FedoryuK, Asymptotic behavior of solutions of $w^{\prime \prime}(z)$ $-p(z, \lambda) w(z)=0$ as $\lambda \rightarrow \infty$ in the complex $z$-plane, Uspehi Mat. Nauk 21 (1966), pp. 3-50.

[3] Fröman, M. AND P. O. Fröman, JWKB-approximation, North-Holland, Amsterdam (1965).

[4] FEDoryuk M.B., Encyclopedia of Mathematical Sciences Vol. 13, Analysis I, edited by R. V. Gamkrelidze, Springer-Verlag (1986), Chapter 2.

[5] Heading, J., An introduction to phase-integral methods, London: Methuen (1962).

[6] Olver, F. W.J., General connection formulae for Liouville-Green approximations in the complex plane, Philosophical Transactions of the Royal society of London, 289 (1978), 501-548.

[7] PARIS R.B. AND A.D. Wood, Asymptotics of high order differential equations, Longman Scientific \& Technical (1986).

[8] Voros, A., The return of the quartic oscillator, The complex WKB method. Ann. Inst. Henri Poincare, 39 (1983), pp. 211-338.

Department of Mathematics, Keio University

HiYoshi 3-14-1, YoKoHAMA, Kanagawa 223, JaPAN

Division of General Education, TaKachiHo University

Ohmiya 2-19-1, Suginami-ku, Tokyo 168 , Japan

Department of Mathematics, Tokyo Institute of Technology

O-OKAyama 2-12-1, Meguro-Ku, TOKYo 152, JaPAN 